\title{
Assessing road network vulnerability: a User Equilibrium interdiction model
}

\author{
Stefano Starita ${ }^{a}$ and Maria Paola Scaparra ${ }^{b}$ \\ aSirindhorn International Institute of Technology, Thammasat University, Pathum Thani, \\ Thailand; ' Kent Business School, University of Kent, CT2 7PE Canterbury, UK
}

\begin{abstract}
ARTICLE HISTORY
Compiled September 24, 2019

ABSTRACT

Road networks are vulnerable to natural and man-made disruptions. The loss of one or many critical links of the network often leads to increased traffic congestion. Therefore, quantitative models are necessary to identify these critical assets so that actions can be taken by decision makers to mitigate the impact of disruptions. This paper proposes an optimisation model to identify the set of arcs that, when lost, results in the worst congestion under user equilibrium traffic. The model is formulated as a bi-level non-linear problem. The challenging formulation is solved via a customised version of Greedy Randomised Adaptive Search Procedure (GRASP) meta-heuristic. Computational experiments are run on a dataset of artificial grids and managerial insights are provided based on popular Sioux and Berlin network case-studies.
\end{abstract}

\section{KEYWORDS}

Road Vulnerability; Critical Infrastructure; Disruption Management; Interdiction Model; User Equilibrium

\section{Introduction}

Transportation networks are the backbone of every country and due to their importance to the well-being of communities and societies are considered Critical Infrastructure. Nowadays, commodities are transported mostly on the road via trucks. This is particularly true if we focus on local transportation or transportation within neighboring countries. In 2017 more than $65 \%$ of US-NAFTA merchandise was carried by trucks. Rail lagged well behind with about $15 \%$. Other transportation modes (air, vessel, pipeline) carried less significant shares of commodities (Bureau of Transportation Statistics, 2017a). Similarly, in the UK about $76 \%$ of goods are moved by road (Department for Transport, 2016). Road is generally the main transportation mode also when it comes to move people. Again, looking at the US, between 2000 and 2015 almost $90 \%$ of journeys to work were made using personal vehicles (Bureau of Transportation Statistics, 2017b) whereas in the UK in 2015 about $64 \%$ of trips were covered by cars. Developing countries are even more vulnerable to road infrastructure disruptions as they often lack alternative transportation modes. As a consequence, disruptive events can render these countries incapable of accommodating the entire flow of people and 
commodities.

Because of this critical socio-economical role, disruptions of road networks often take a high economic toll. During 2015-2016, the UK was hit by intense rainfall leading to widespread flooding. The welfare cost of road disruption is estimated to be roughly a hundred million pounds. Focusing on the UK road network, the challenges road transportation is facing are evident. In fact, approximately only $2 \%$ of UK roads accounts for more than $30 \%$ of the total traffic. This clearly indicates that some parts of the network are more critical than others. For example, in 2016 serious floodings led to the closure of main road A591 in Cumbria, disrupting almost 7000 trips a day, for about 6 months (Environmental Agency, 2018). Currently, the UK Environmental Agency estimates that $10 \%$ of the UK road network is vulnerable to flooding.

Particular care in the management of this infrastructure must be applied by countries prone to natural events. Vulnerability analysis has to be implemented also in the wake of climate changes which are bound to generate extreme events more frequently (NASA).

In addition to weather related disruptions, roads are also vulnerable to small scale events such as car accidents, leading to road closure and consequent impact on travel times.

When it comes to road management, quantitative methods are frequently implemented to rank assets of the infrastructure according to some vulnerability metrics. These rankings are then used to identify the most critical assets of the infrastructure. The information is then used by decision makers to build contingency plans or design network enhancements. This paper contributes to the body of research devoted to assess vulnerability in a road transportation network. An optimisation model is introduced to identify the most vulnerable components in a road network under risk disruption. We focus on strategic risk (Golany et al., 2009) by modeling worst-case disruptions. This approach should be considered as complementary to the probabilistic risk one. Decision makers require both perspectives when budget allocation decisions have to be made and people's livelihood is at stake. To better capture the impact of disruptions on the system's congestion, the road network is assumed to be a User Equilibrium (UE) network. Every driver aims to minimise his own trip's cost and the UE is achieved once no driver can reduce his cost via unilateral actions. This is also known as the Wardrop's first principle (Wardrop, 1952).

The remainder of the paper is organized as follows: Section 2 provides an in-depth review of the relevant literature. In Section 3, the optimisation model is introduced and described. Section 4, focuses on the algorithmic approach used to efficiently solve the complex formulation. A computational analysis of the algorithm is implemented using grids of different sizes. Section 5, applies the model to Sioux fall and Belrin Friedrichshain case-studies to provide an example on how the results can be used to support decision-making. Finally, conclusions and future developments are discussed in Section 6.

\section{Background}

Research on disruption management for Critical Infrastructure is vast and keeps evolving at a fast pace. That is particularly true for both supply-chain and transportation infrastructure. The problem can be inspected from different angles. For example, some researchers have focused on the design stage, to build infrastructure that are inher- 
ently resilient (Cadarso and Marín, 2016; Laporte et al., 2011; Martel et al., 2013; Tran et al., 2017). Focusing on road networks, design models have been studied investigating aspects such as robustness and resiliency in presence of different types of uncertainties. For instance, Ukkusuri et al. (2007) study the problem under stochastic demand. They propose a Genetic Algorithm to minimise the expected travel costs together with standard deviation across scenarios. Dimitriou et al. (2008) propose a reliable design model to hedge against uncertainty in demand, supply and drivers' behavior. Kepaptsoglou et al. (2014) shift the focus to the post-disruption phase by simultaneously deciding on highway design and recovery operations so as to maximise the performance of the surviving system. A comprehensive review of papers studying resiliency of transportation systems can be found in Mattsson and Jenelius (2015).

In this paper, we assume the transportation system to be pre-existent and we focus on the pre-disaster stage.

This section focuses on papers that develop quantitative approaches to assess the vulnerability of a road system. Normally, the road system is modeled as a network of nodes and arcs, hence we broaden the scope of the analysis to network vulnerability in general.

Within this scope, two broad categories of approaches to assess vulnerability can be identified: vulnerability metrics and mathematical optimisation models.

\subsection{Vulnerability metrics}

This line o fresearch focuses on identifying vulnerability metrics to rank the different assets of a network. The usual approach is to measure the system's performance in a normal state and in a post-disruption state after an asset (or sometimes a group of assets) is inoperable. The vulnerability of the network toward that asset is measured in terms of the delta between pre and post-disruption states. Within this framework, the main difference among research papers is how the system's performance is measured. Many have developed structure-based metrics whereby vulnerability is assessed as a function of features such as connectivity, shortest path, node-betweenness etc. For example, Wang and Rong (2009) introduce a node-degree driven metric for a power grid vulnerable to cascade attacks. Yin and Xu (2010) compute the efficiency of Shanghai road network as the sum of the recripocal of the shortest paths. The vulnerability of Beijing railway transit network is evaluated by Sun et al. (2018) in terms of nodedegree, betweenness and strength.

Another transportation feature that is frequently embedded into vulnerability analysis is the accessibility. The concept of accessibility is modeled in different ways but generally is built around socio-economical and behavioral criteria. In Sohn (2006), accessibility indices are computed for each county using factors such as population, shortest path, average traffic etc. Taylor et al. (2006) use the Hansen integral index which defines accessibility as a function of the attractiveness of a location. The same index is used by Luathep et al. (2011) in a large-scale vulnerability analysis study applied to the Bangkok metropolitan area. Taylor and Susilawati (2012) propose a metric for regional and remote areas based on the concept of remoteness, defined as the inverse of the accessibility.

A further approach more closely related to road transportation exploits concepts such as flow or congestion to build vulnerability metrics. For example, Jenelius et al. (2006) rank arcs and nodes of a road system by building two metrics called importance and exposure. These metrics are based on the increase of the travel cost when an ele- 
ment is inoperable. Scott et al. (2006) argue that metrics based on the volume/capacity ratio fail to capture system-wide impacts and introduce the Network Robustness Index. This index is computed by evaluating the User Equilibrium of the network after removing one arc. The same approach is used by Sullivan et al. (2010) to build a system-wide metric called Network Trip Robustness. These approaches are extended by Jenelius and Mattsson (2012), where the focus is on disruption scenarios where spatially correlated arcs are simultaneously inoperable. Watling and Balijepalli (2012) develop a moment-based method to compute vulnerability in terms of travel times while also taking into account the potential impact of demand growth. El-Rashidy and Grant-Muller (2014) use fuzzification and an exhaustive search optimisation to aggregate different metrics based on both physical properties (length and number of lanes) and network flow. Jin et al. (2017) focus on non-structural disruptions, i.e. events that lead to a capacity reduction for an arc rather than a total closure. Kermanshah and Derrible (2017) measure robustness of Chicago and New York road networks to extreme flooding by comparing the number of pre and post-disruption trips. Leng et al. (2018) build a metric based on a generalised travel cost accounting for vehicle cost, travel time, and traveller comfort.

\subsection{Optimisation models}

Single disruption approaches are generally not too cumbersome from a computational point of view and can be applied to large networks. However, they can be misleading when multiple correlated and uncorrelated disruptions are considered as they cannot accurately capture the system-wise implications of complex disruption scenarios. Events like strikes (Yahoo News, 2016) or floods (Lansing State Journal, 2019) can result in the disruption of multiple segments of a road network. Therefore, when the focus is on larger disruptive events, mathematical optimisation models are best suited (Starita et al., 2017a). Many researchers have investigated the problem of identifying road criticalities and planning for road enhancements assuming a probabilistic risk (Faturechi and Miller-Hooks, 2014; He and Liu, 2012; Ishfaq, 2013; Starita et al., 2017b). Within this stream, several works have studied approaches to measure and optimise robustness and resilience of the network. For example, Chen and Miller-Hooks (2012) propose a mixed-integer formulation to assess the recovery capabilities of a road system under capacity and travel time uncertainty.

In this work we focus on worst-case scenario models instead. These models allow one to identify the most critical arcs with respect to a given performance criterion, such as congestion. A worst-case scenario is usually modelled as a game between two actors with opposite aims: the Interdictor (or attacker), is a proxy to identify most critical assets. It uses a limited amount of resources to inflict the highest damage to the system (for instance, by disrupting a number of arcs). The User minimises/maximises the system's cost/benefit. This game is often formulated as a bi-level optimisation model (Brown et al., 2006) and referred to as Interdiction problem. Research in this area has gained momentum in the past 15 years with the focus being primarly on supply-chain infrastructure (Akbari-Jafarabadi et al., 2017; Aksen et al., 2013; Church et al., 2004; Losada et al., 2012).

Building on the seminal paper by Richard Wollmer (1964), a number of studies have appeared studying the interdiction problem on flow-based transportation networks. Wood (1993) and Kelly J. et al. (1998) propose mixed-integer formulations for the deterministic and stochastic max-flow interdiction problem. Different formu- 
lations and solution approaches are considered by Murray et al. (2007), Myung and Kim (2004), Lunday and Sherali (2012), and Soleimani-Alyar and Ghaffari-Hadigheh (2018). Afshari Rad and Kakhki (2013) add the temporal component to the problem along with a solution algorithm based on Benders Decomposition. Rad and Kakhki (2017) focus on the computational challenge of the model by providing an efficient re-formulation and a set of valid inequalities.

Focusing on distance-based networks, the shortest-path interdiction problem is introduced by Fulkerson and Harding (1977) and later on formulated as a mixed-integer problem by Israeli and Wood (2002). Wei et al. (2018) extend the shortest-path interdiction with the aim of finding the minimum resources needed to increase the path length to a given threshold. Bidgoli and Kheirkhah (2018) propose a vehicle-routing interdiction problem to inspect how logistics profits are vulnerable to arc disruptions. They also assume asymmetry of information between interdictor and user.

The models described above are suitable for many infrastructure systems, however they do not take into account the congestion and drivers behavior typical of a road infrastructure. For example, a model based on a shortest path can be applied to a road network only under the strong assumption that every arc is free of congestion and drivers always choose the shortest path. Typical features of road transportation systems are better captured by Traffic Assignments models that aim to compute the so-called User Equilibrium (UE). Mathematical formulations and algorithms have been proposed to find the UE, which is a traffic flow assignments that satisfy the Wardrop's first principle. Specifically, at UE the travel time on all used paths is equal, and (also) less than or equal to the travel time that would be experienced by a single vehicle on any unused path (Sheffi, 1985). In other words, at equilibrium, no driver can individually decrease its travel effort by choosing a new route. To the best of these authors' knowledge, the interdiction problem on a User Equilibrium network has not been investigated so far. Numerous papers investigate the problem of road network design problem (Bruynooghe, 1972; Poorzahedy and Turnquist, 1982) which can be formulated using a similar bi-level structure to our interdiction problem. However, the model introduced in this paper is applied to a different context, with the objective of informing decision makers about critical hot-spots of the network and where to invest to mitigate the impact of disruptions. To summarise, the main contributions of these paper are:

- Formulating the Interdicion problem on User Equilibrium networks

- Introducing a GRASP inspired heuristic to efficiently solve the problem

- Presenting a case study on the Sioux and Berlin networks to illustrate how the model can be used to inform decision-makers.

\section{A User Equilibrium based interdiction model}

\subsection{Mathematical formulation}

This section introduces the formulation for the bi-level UE interdiction model. We focus on a road network, modelled as a directed, weighted graph $G(N, A)$, where $N$ is the set of nodes and $A$ the set of arcs. Traveling time on a given $\operatorname{arc} a$ is a function $t_{a}()$ of the traffic flow on that arc. The objective of the model is to identify the set of arcs that, when disrupted, would yield the highest system's cost. In our problem, the system's cost is measured as the product of traffic flows and travel times. The model takes into account the impact of congestion by computing the user equilibrium 
in the inner level. The outer level models the actions of the interdictor whose aim is to maximise the system's cost. We assume that no traffic is allowed on a disrupted arc and the interdictor can disrupt up to $R$ arcs. The overall model's notation is summarized below:

Sets, indexes and parameters:

- $N$ is the set of nodes

- $A$ is the set of links

- $r, s$ are indexes used for nodes

- $a$ is the index used for links

- $K_{r s}$ is the set of paths connecting origin $r$ to destination $s$

- $k$ is the index used for paths

- $q_{r s}$ is the trip rate between origin $r$ and destination $s$

- $R$ is the number of disrupted links

- $\delta_{a k}^{r s}$ is a binary parameter equal to 1 if arc $a$ belongs to path $k$, connecting origin $r$ to destination $s ; 0$ otherwise

- $t_{a}=t_{a}\left(x_{a}\right)$ is the actual travel time of a link $a$, defined as a function of the traffic flow on the link.

Decision variables:

- $x_{a}$ is the flow on link $a$

- $\omega$ auxiliary flow variable used for integration

- $f_{k}^{r s}$ is the flow on path $k$ connecting origin $r$ to destination $s$

- $y_{a}$ is the interdiction variable equal to 1 if $\operatorname{arc} a$ is disrupted; 0 otherwise

The User Equilibrium Interdiction Model (UEIM) is formulated as follows:

$$
\begin{aligned}
& \text { [UEIM] } \underset{\mathbf{y}}{\operatorname{maximize}} \sum_{a \in A} x_{a} t_{a}\left(x_{a}\right) \\
& \text { subject to } \quad \sum_{a \in A} y_{a} \leq R \\
& y_{a} \in\{0,1\} \quad \forall a \in A \\
& \operatorname{minimize}_{\mathbf{x}, \mathbf{f}} \sum_{a \in A} \int_{0}^{x_{a}} t_{a}(\omega) d \omega \\
& \text { s.t. } \quad \sum_{k \in K_{r s}} f_{k}^{r s}=q_{r s} \forall r \in N, \forall s \in N \backslash\{s\} \\
& x_{a}=\sum_{r \in N} \sum_{s \in N \backslash\{r\}} \sum_{k \in K_{r s}} f_{k}^{r s} \delta_{a k}^{r s} \quad \forall a \in A \\
& f_{k}^{r s} \geq 0 \quad \forall r \in N, \forall s \in N \backslash\{s\}, \forall k \in K_{r s} \\
& x_{a} \leq M\left(1-y_{a}\right) \quad \forall a \in A
\end{aligned}
$$

The upper level model (1)-(3) represents the interdiction problem. The aim of the objective function (1) is to maximise the total system's cost expressed as the product of travel time and flow. Inequality (2) is the cardinality constraint on the maximum number of disrupted arcs. Constraints (3) are the binary requirements for variables $y_{a}$.

The lower level model (4)-(7) is a standard User Equilibrium problem. The objective 
function (4) is used to compute the equilibrium flows of the network. Constraints (5) state that the entire demand is to be served. Constraints (6) compute the total flow traversing link $a$. Constraints (7) are non-negativity requirements for variables $f_{k}^{r s}$. Finally, the set of inequalities (8) enforce that no traffic is allowed on a disrupted arc.

\subsection{Solution approach}

Solving UEIM is generally difficult because of its bi-level structure. In fact, linear and non-linear bi-level programs have been proven to be NP-hard (Wiesemann et al., 2013). The complexity of this type of problems has been widely discussed for the transportation network design problem, where the role of the interdictor is replaced by a network designer whose aim is to expand the network, given a limited budget. Within this context many researchers have recurred to heuristic and meta-heuristic approaches (Drezner and Wesolowsky, 2003; Kim and Kim, 2006; Poorzahedy and Abulghasemi, 2005).

In this section, we propose a solution algorithm as a customised version of the Greedy Randomised Adaptive Search procedure (GRASP) metaheuristic (Feo and Resende, 1995). As in GRASP, the algorithm starts with an iterative step and at each stage builds a solution by adding elements from a dynamic candidate set. The solution is further improved via a search following the initial step. However, the algorithm is different from standard GRASP in that it generates a list of best solutions, not just the best found. Furthermore, driven by preliminary results, the standard local search is replaced by a simple single-swap applied to the solutions of the list. Overall, we refer to this algorithm as Multi-Start GRASP (MSG), since it basically consists in re-starting the GRASP procedure a number of times, while keeping the best solutions. A detailed description of the building blocks of the algorithm is given below.

\subsubsection{Multi-Start GRASP (MSG) procedure}

The algorithm builds tentative solutions by randomly choosing arcs to disrupt from a dynamic candidate set. This set is updated at each stage so that it contains the most promising arcs. The key decision is how to quantify the concept of promising from the interdictor's perspective. To this aim, the algorithm uses variables $x_{a}$ based on the simple rationale that arcs that intercept higher flows are more likely to increase the congestion of the system, when disrupted. In order to describe the pseudo-code (shown in Algorithm1), we need to introduce some further notation. Let $I$ be a dynamic list keeping the best $p$ solutions discovered, $C S$ be the candidate set with the most promising arcs and $\mathrm{UE}(\mathbf{y})$ be the User Equilibrium problem for a given interdiction vector $\mathbf{y}$.

At each iteration $\tau$, the algorithm starts with an empty disruption set. The solution is built by adding $R$ arcs in sequence. To build the candidate set $C S$, the user equilibrium problem is solved for the given $\mathbf{y}^{\tau}$. After a solution is built (i.e., $R$ arcs have been selected by setting the $y$ variables to 1 , accordingly), a final user equilibrium is solved to compute the total system's cost. This cost is then used to evaluate the solution and decide if it should be stored or discarded. The algorithm ends after a pre-defined number of iterations and returns the list of $p$-best solutions found. 


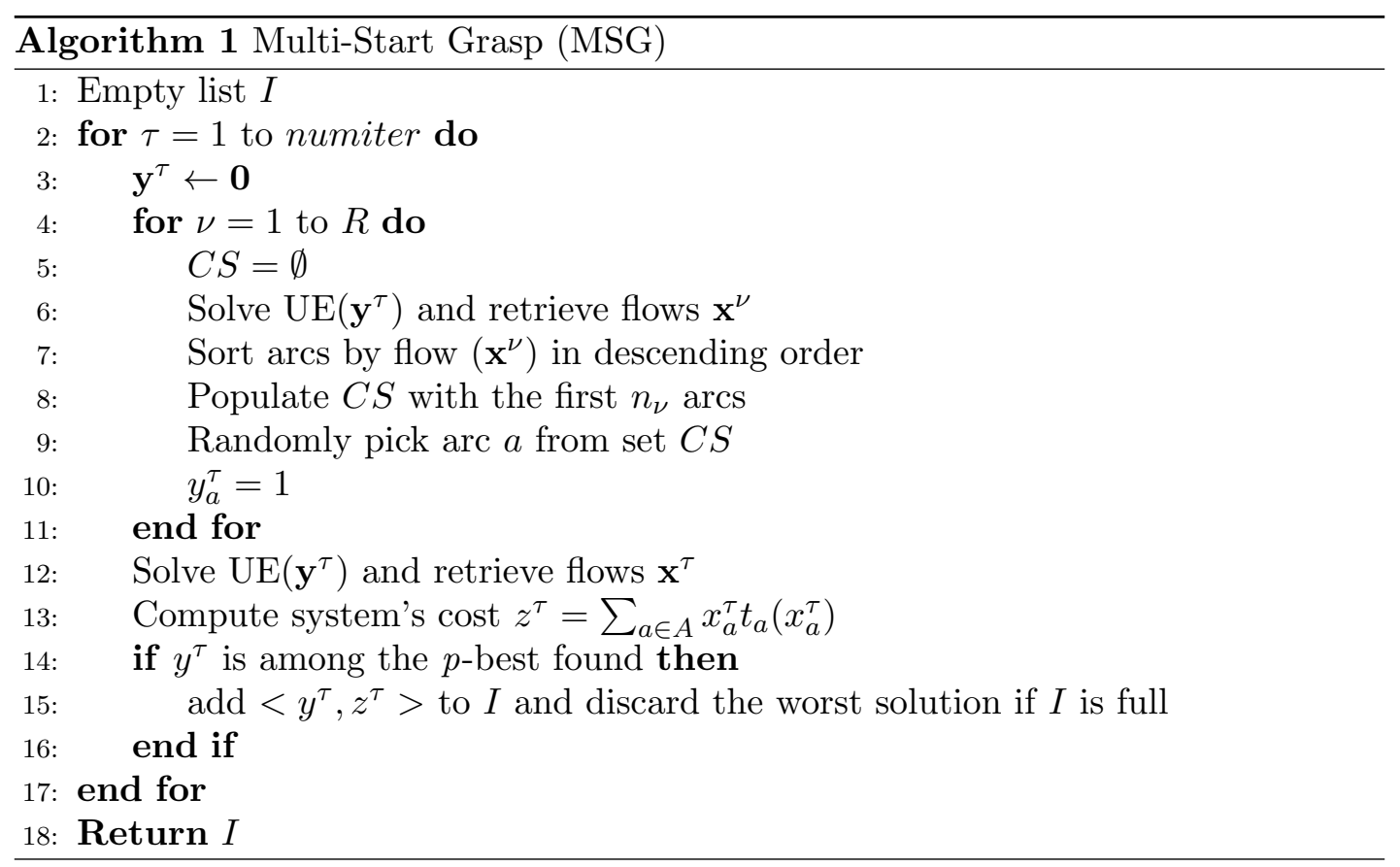

\subsubsection{Single-Arc Swap (SAS) procedure}

This search procedure is implemented to further improve the solutions generated by the MSG step. The search performs an enumeration of the one-to-one swaps, replacing one arc in the solution with another arc from the neighborhood. The pseudo-code is described in Algorithm 2.

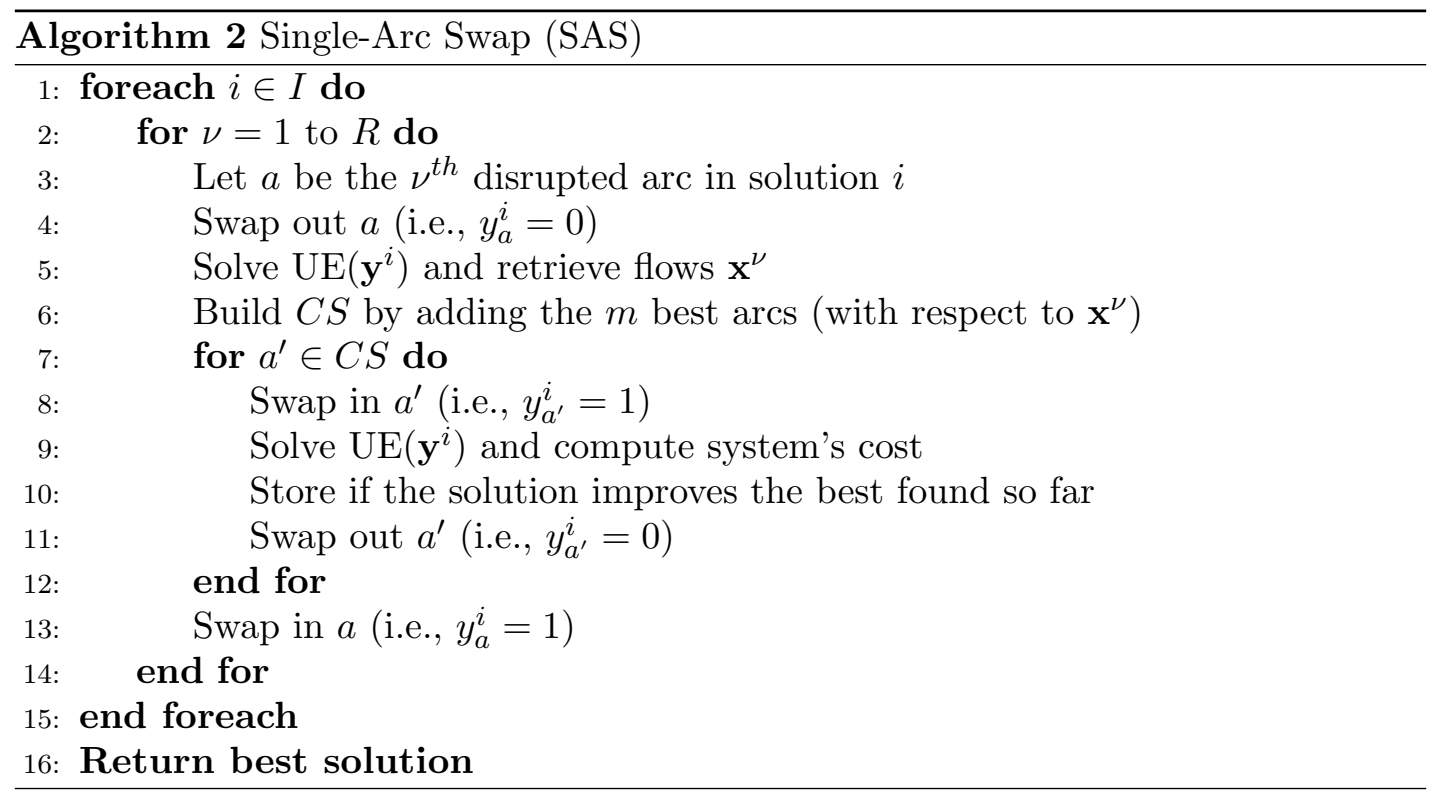

The starting point of this search is the set of best solutions obtained by MSG and stored in list $I$. The search is performed for every solution in $I$. For a given solution $i \in I$, disrupted arcs are individually swapped out and a full search of the neighborhood is executed. Assuming $a$ to be the arc swapped out from the initial solution (line 4), 
the neighbourhood is identified by solving $\mathrm{UE}\left(\mathbf{y}^{i}\right)$ and populating $C S$ with the $m$ best solutions according to the resulting flow variables. All arcs in $C S$ are sequentially swapped-in to create a new solution. The system's cost of each solution is computed by solving the User Equilibrium problem (line 9). The best solution is stored and returned after the end of the search.

\subsubsection{Solving the User Equilibrium problem}

Solving the bi-level problem necessarily requires an efficient algorithm for the user equilibrium subproblem. This problem has been studied in-depth for decades and the literature is rich with exact and approximate algorithms to compute the network equilibrium. Expanding the literature on this topic is beyond the scope of this paper. We simply implement the convex algorithm defined in the seminal work by Sheffi (1985), which is one of the most efficient algorithms to solve UE problems and quickly converges to the equilibrium solutions.

\section{Computational experiments}

This section provides a computational analysis of the solution approaches introduced before. The algorithms are written in $\mathrm{C}++$ using Visual Studio and are run on a PC with AMD Ryzen 71700 and 8GB RAM.

\subsection{Instances generation}

A set of artificial networks of different sizes is created to assess the accuracy and scalability of the solution approaches. These networks are built as square grids (Fig. 1). Although artificially generated, these grids replicate the structure of many postindustrial cities that are developed around the concept of city-block (Blumenfeld, 1949). Furthermore, grids can be easily scaled up, for this reason are frequently used to build dataset to evaluate algorithms (Bekhor and Toledo, 2005; Chiou, 2005; Zhang et al., 2014). Popular Bureau of Public Roads (BPR) formula is used to formulate the relationship between travel times and traffic flows. Specifically, $t_{a}\left(x_{a}\right)=\bar{t}_{a}\left(1+\alpha\left(\frac{x_{a}}{c_{a}}\right)^{\beta}\right)$, where $\bar{t}_{a}$ is the free-flow travel time (i.e., travel time on $a$ when there is no congestion), $c_{a}$ the nominal capacity of link $a$ and $\alpha$ and $\beta$ some traffic parameters. 


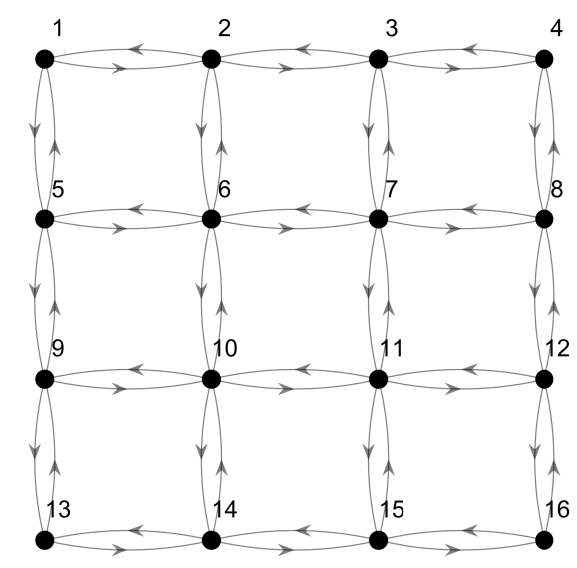

Figure 1. 16-nodes grid

While keeping the same network structure, the rest of the problems parameters are changed across instances as indicated in Table 1. Values have been chosen looking at past literature.

Table 1. Instances parameters

\begin{tabular}{cc}
\hline Parameters & Choices \\
\hline$\alpha$ & 0.15 \\
$\beta$ & 4 \\
$c_{a}$ & $1500,3000,4500$ \\
$t_{a}$ & $4,8,12$ \\
\hline
\end{tabular}

The generation of the traffic demand requires a more detailed discussion. In order to generate meaningful demand patterns in the networks, we build the $q_{r s}$ parameters using a gravity model. The gravity model is used to reflect in the demand that areas with higher population will generate and attract larger number of trips. To this aim, a population pop $_{i}$ of 300, 450 or 600 is randomly assigned to each node $i$. These values are set so that the smallest demand zone will have half the population of the largest one. Distances between nodes are then computed solving an all-pairs shortest path problem (assuming free flow). Finally, the demand between any $r$ and $s$ is obtained as the ratio between the product of respective populations and the square of their distance, in accordance with the gravity model.

The population numbers are set purposely high so that the resulting networks are always highly congested. To generate instances with different levels of congestion, we run a scaling-down procedure with the following constraints:

$$
\frac{1}{|A|} \sum_{a \in A} \frac{x_{a}}{c_{a}} \leq T_{a v}^{c o n g}
$$




$$
\frac{x_{a}}{c_{a}} \leq T_{\max }^{c o n g}, \quad \forall a \in A
$$

The first constraint is an upper bound on the average congestion, whereas the second constraint limits the maximum congestion accepted on each link. The scaling-down iterative procedure is described by Algorithm 3:

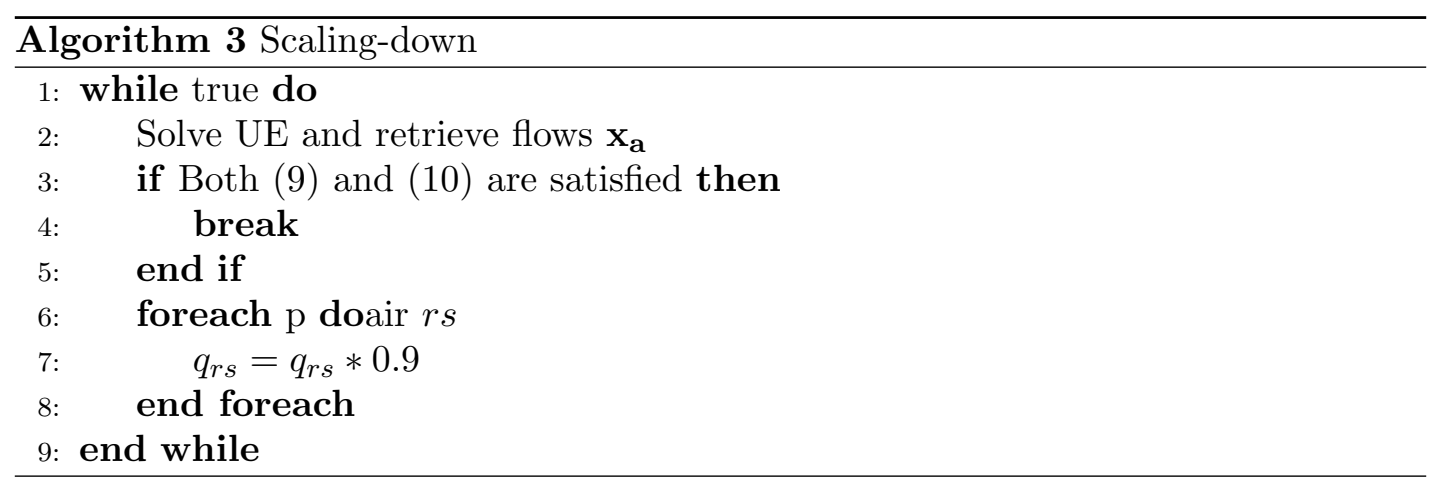

The algorithm iteratively decreases the original flow demand by $10 \%$ until the requirements on congestion are met. We generate a dataset made of congested networks $\left(T_{a v}^{c o n g}=0.8\right.$ and $\left.T_{m a x}^{c o n g}=1.5\right)$ and heavily-congested networks $\left(T_{a v}^{c o n g}=1.2\right.$ and $\left.T_{\max }^{c o n g}=2.5\right)$. The latter are identified by adding $h$ as a suffix in their name. This categorization is based under the common assumption that a link is congested once the number of cars exceeds its capacity. Furthermore, we add to the dataset the popular Sioux and the Berlin Friedrichshain networks to analyse the problem on different network structures and provide insights on case studies that have been frequently investigated in the past. The case studies are downloaded from the online repository "Transportation Networks for Research" and their parameters are set according to Leblanc (1975) (Sioux network) and Jahn et al. (2005) (Berlin network). A summary of some structural features of the networks is given in Table 2. Note that Zones is used to refer to nodes that generate and attract demand. This is used to differentiate nodes that could simply represent intersections. In the artificial grids and Sioux network every node is also a demand point, whereas out of the 361 nodes of Berlin network only 26 are demand points. The table also list the average shortest paths and a measure of transitivity computed as the ratio between the number of three-arcs loops and two-arcs paths.

Table 2. Instances structural features

\begin{tabular}{ccccccc}
\hline Name & Nodes & Arcs & $\begin{array}{c}\text { Demand } \\
\text { Zones }\end{array}$ & OD Pairs & $\begin{array}{c}\text { Average } \\
\text { Shortest Path }\end{array}$ & Transitivity \\
\hline $16-\mathrm{x}$ & 16 & 48 & 16 & 240 & 13 & 0.00 \\
$25-\mathrm{x}$ & 25 & 80 & 25 & 600 & 16 & 0.00 \\
$36-\mathrm{x}$ & 36 & 120 & 36 & 1260 & 18 & 0.00 \\
$64-\mathrm{x}$ & 64 & 224 & 64 & 4032 & 24 & 0.00 \\
$100-\mathrm{x}$ & 100 & 360 & 100 & 9900 & 30 & 0.00 \\
Sioux & 24 & 76 & 24 & 552 & 11 & 0.07 \\
Berlin & 361 & 766 & 26 & 650 & 33 & 0.19 \\
\hline
\end{tabular}




\subsection{Algorithms evaluation}

In this section, results of the computational experience on the dataset are presented. To provide a benchmark for our algorithms, we implement a complete enumeration procedure where all the interdiction strategies are explored to identify the one(s) yielding the highest travel cost. Unfortunately, this approach is applicable only to small problems (i.e., up to 36 nodes). For larger instances, we implement a greedy algorithm (RG) with a random starting point. Specifically, the interdiction set is initialised with a randomly selected arc. Subsequently, the remaining $R-1$ arcs are sequentially chosen using a greedy criterion. Specifically, every arc not yet in the solution is individually added to the interdiction set and the system cost is computed. After all arcs are evaluated, the one yielding the highest cost is permanently added to the solution. Changing the starting arc results in different disruption strategies being evaluated. The algorithm is iterated so that it solves the same number of UE problems as MSGSAS. As for the parameters of MSG-SAS, we allow the size of the $C S$ (i.e., $n_{\nu}$ ) to linearly decrease from 12 , when $\nu=1$ (i.e., the solution being constructed is empty) to 2 when $\nu=4$. This choice is motivated by the observation that as the number of arcs in the solution (identified by $\nu$ ) increases, good candidate arcs are more frequently found in the top ranking positions. The size of the $C S$ (i.e., ) during SAS is set to 4 . The number of iterations is 500 for problems with $R \leq 3,5000$ when $R>3$. Overall, the number of UE calls is mostly defined by the MSG procedure, which needs to solve numiter $(1+R)$ UE problems.

Tables 3, 4, and 5 compare results of the algorithms on small networks. The interdictor budget $R$ is set equal to 2,3 , and 4 . Column $\mathrm{Obj}$, lists the objective function found by the enumeration procedure. Column CPU Time reports the computational time in seconds. Finally, Gap column is the percentage difference between the best solution found by any algorithm and the one found via full enumeration. For network instances with 36 nodes and $R=4$, the full enumeration is no longer computationally feasible. In this cases, the Gap columns, report the gaps between the best objective found by one algorithm and the absolute best objective found across all algorithms. Statistical descriptors such as Average, Median, Interquartile range (IQR), max and min are also reported in bold at the bottom of each table.

The tables show that MSG-SAS always return a 0\% gap against the Enumeration algorithm. This is a positive outcome as it is clear that a full enumeration approach does not scale with the size of a problem and the number of disrupted arcs and, therefore, can only be used for small examples. Results also highlight that RG performances are inconsistent as it sometimes finds good solutions but also return gaps as high as $82.9 \%$. Its average gap ranges between $13 \%$ and $28 \%$. However, in one occasion (36-1 and $R=4$ ) RG outperforms MSG-SAS by $1.9 \%$. The accuracy of MSG worsen when $R$ increases from 2 to 3 , with respective average gap growing from $5 \%$ to $19 \%$. The lower average gap reported in Table 5 is due to the above mentioned impossibility of obtaining solutions by full enumeration with 36-node networks. With the sole exception of instance 36-1, the best solution is always found by MSG-SAS.

Looking at the computational times, the advantage of MSG-SAS against the full enumeration is particularly evident with large instances and $R>2$. For example, when $R=3$, MSG-SAS is on average two orders of magnitude faster and when $R=4$ the enumeration cannot be implemented for 36 -node networks as it would require to solve $\left(\begin{array}{c}|A| \\ R\end{array}\right) \sim 8 \mathrm{M}$ user equilibrium problems.

Given the randomness of the MSG procedure, it is worth investigating how robust MSG and MSG-SAS performances are. To this aim, we perform a further analysis 
Table 3. Comparison of solution approaches when 2 arcs are disrupted $(R=2)$

\begin{tabular}{|c|c|c|c|c|c|c|c|c|}
\hline \multirow[b]{2}{*}{$\begin{array}{l}\text { Instance } \\
\text { Name }\end{array}$} & \multicolumn{2}{|c|}{ Enumeration } & \multicolumn{2}{|c|}{$\mathrm{RG}$} & \multicolumn{2}{|c|}{ MSG } & \multicolumn{2}{|c|}{ MSG-SAS } \\
\hline & Obj & $\begin{array}{l}\text { CPU } \\
\text { Time(s) }\end{array}$ & $\operatorname{Gap}(\%)$ & $\begin{array}{l}\text { CPU } \\
\text { Time(s) }\end{array}$ & $\operatorname{Gap}(\%)$ & $\begin{array}{l}\text { CPU } \\
\text { Time(s) }\end{array}$ & $\operatorname{Gap}(\%)$ & $\begin{array}{l}\text { CPU } \\
\text { Time(s) }\end{array}$ \\
\hline $16-1$ & 2204.2 & 1.1 & $34.2 \%$ & 2.3 & $0.0 \%$ & 1.6 & $0.0 \%$ & 2.2 \\
\hline $16-2$ & 4950.1 & 1.1 & $1.8 \%$ & 2.4 & $0.0 \%$ & 1.6 & $0.0 \%$ & 2.4 \\
\hline $16-3$ & 2258.8 & 1.3 & $0.0 \%$ & 2.7 & $0.0 \%$ & 1.9 & $0.0 \%$ & 2.6 \\
\hline $16-4$ & 2103.5 & 0.9 & $17.1 \%$ & 1.6 & $0.0 \%$ & 1.2 & $0.0 \%$ & 1.7 \\
\hline $25-1$ & 1935.7 & 7.3 & $0.0 \%$ & 5.2 & $0.0 \%$ & 3.6 & $0.0 \%$ & 5.3 \\
\hline $25-2$ & 2411.2 & 8.2 & $28.1 \%$ & 5.8 & $0.0 \%$ & 3.6 & $0.0 \%$ & 5.5 \\
\hline $25-3$ & 2246.7 & 8.7 & $44.1 \%$ & 6.1 & $6.8 \%$ & 3.9 & $0.0 \%$ & 5.5 \\
\hline $25-4$ & 1700.5 & 9.4 & $0.0 \%$ & 8.1 & $0.0 \%$ & 4.3 & $0.0 \%$ & 6.2 \\
\hline $36-1$ & 2544.4 & 40.5 & $21.2 \%$ & 12.5 & $11.5 \%$ & 8.9 & $0.0 \%$ & 13.1 \\
\hline $36-2$ & 21758.5 & 82.5 & $54.6 \%$ & 25.2 & $0.0 \%$ & 19.5 & $0.0 \%$ & 29.3 \\
\hline $36-3$ & 12631.1 & 68.9 & $39.4 \%$ & 20.5 & $0.0 \%$ & 15.6 & $0.0 \%$ & 24.0 \\
\hline $36-4$ & 1823.6 & 39.5 & $5.7 \%$ & 12.1 & $0.0 \%$ & 9.1 & $0.0 \%$ & 13.5 \\
\hline $16-1 \mathrm{~h}$ & 17839.6 & 1.9 & $0.0 \%$ & 4.0 & $0.0 \%$ & 2.7 & $0.0 \%$ & 4.0 \\
\hline $16-2 \mathrm{~h}$ & 14112.7 & 2.2 & $0.0 \%$ & 4.6 & $0.0 \%$ & 3.0 & $0.0 \%$ & 4.6 \\
\hline $16-3 \mathrm{~h}$ & 39762.6 & 1.8 & $0.0 \%$ & 3.8 & $0.0 \%$ & 2.8 & $0.0 \%$ & 4.3 \\
\hline $16-4 \mathrm{~h}$ & 9292.1 & 2.5 & $0.0 \%$ & 5.1 & $6.4 \%$ & 3.3 & $0.0 \%$ & 5.0 \\
\hline $25-1 \mathrm{~h}$ & 32341.9 & 15.6 & $0.0 \%$ & 11.1 & $0.0 \%$ & 8.1 & $0.0 \%$ & 12.3 \\
\hline $25-2 \mathrm{~h}$ & 29647.7 & 15.1 & $62.9 \%$ & 10.9 & $0.0 \%$ & 7.5 & $0.0 \%$ & 11.2 \\
\hline $25-3 \mathrm{~h}$ & 6621.8 & 15.5 & $26.8 \%$ & 10.6 & $0.0 \%$ & 8.8 & $0.0 \%$ & 13.2 \\
\hline $25-4 \mathrm{~h}$ & 27179.5 & 14.2 & $0.0 \%$ & 10.2 & $0.0 \%$ & 7.2 & $0.0 \%$ & 10.8 \\
\hline $36-1 \mathrm{~h}$ & 28511.2 & 107.3 & $64.0 \%$ & 33.6 & $64.0 \%$ & 27.9 & $0.0 \%$ & 40.5 \\
\hline $36-2 \mathrm{~h}$ & 15387.6 & 87.5 & $31.4 \%$ & 26.2 & $0.0 \%$ & 20.9 & $0.0 \%$ & 32.3 \\
\hline $36-3 h$ & 16388.5 & 102.7 & $0.0 \%$ & 31.4 & $25.2 \%$ & 22.8 & $0.0 \%$ & 35.2 \\
\hline $36-4 h$ & 12977.8 & 80.8 & $28.1 \%$ & 25.0 & $16.6 \%$ & 19.1 & $0.0 \%$ & 31.0 \\
\hline Sioux & 2663.6 & 5.2 & $0.0 \%$ & 3.9 & $0.0 \%$ & 2.6 & $0.0 \%$ & 3.8 \\
\hline Sioux-h & 29692.2 & 21.0 & $0.0 \%$ & 19.4 & $0.0 \%$ & 12.1 & $0.0 \%$ & 18.2 \\
\hline Average & & 28.6 & $17.7 \%$ & 11.7 & $5.0 \%$ & 8.6 & $0.0 \%$ & 13.0 \\
\hline Median & & 11.8 & $3.7 \%$ & 9.2 & $0.0 \%$ & 5.8 & $0.0 \%$ & 8.5 \\
\hline IQR & & 45.5 & $32.1 \%$ & 15.7 & $1.6 \%$ & 10.2 & $0.0 \%$ & 15.4 \\
\hline Min & & 0.9 & $0.0 \%$ & 1.6 & $0.0 \%$ & 1.2 & $0.0 \%$ & 1.7 \\
\hline Max & & 107.3 & $64.0 \%$ & 33.6 & $64.0 \%$ & 27.9 & $0.0 \%$ & 40.5 \\
\hline
\end{tabular}


Table 4. Comparison of solution approaches when 3 arcs are disrupted $(R=3)$

\begin{tabular}{|c|c|c|c|c|c|c|c|c|}
\hline \multirow[b]{2}{*}{$\begin{array}{l}\text { Instance } \\
\text { Name }\end{array}$} & \multicolumn{2}{|c|}{ Enumeration } & \multicolumn{2}{|c|}{ RG } & \multicolumn{2}{|c|}{ MSG } & \multicolumn{2}{|c|}{ MSG-SAS } \\
\hline & Obj & $\begin{array}{l}\text { CPU } \\
\text { Time(s) }\end{array}$ & $\operatorname{Gap}(\%)$ & $\begin{array}{l}\text { CPU } \\
\text { Time(s) }\end{array}$ & $\operatorname{Gap}(\%)$ & $\begin{array}{l}\text { CPU } \\
\text { Time(s) }\end{array}$ & $\operatorname{Gap}(\%)$ & $\begin{array}{l}\text { CPU } \\
\text { Time(s) }\end{array}$ \\
\hline $16-1$ & 10930.8 & 17.1 & $51.0 \%$ & 3.1 & $0.0 \%$ & 2.2 & $0.0 \%$ & 3.0 \\
\hline $16-2$ & 28775.6 & 18.5 & $22.8 \%$ & 3.7 & $13.3 \%$ & 2.4 & $0.0 \%$ & 3.4 \\
\hline $16-3$ & 12904.1 & 19.8 & $0.0 \%$ & 3.4 & $0.0 \%$ & 2.7 & $0.0 \%$ & 3.7 \\
\hline $16-4$ & 8925.2 & 13.7 & $0.0 \%$ & 2.6 & $0.0 \%$ & 1.8 & $0.0 \%$ & 2.4 \\
\hline $25-1$ & 12051.5 & 190.1 & $69.7 \%$ & 7.9 & $11.8 \%$ & 5.3 & $0.0 \%$ & 8.1 \\
\hline $25-2$ & 10463.0 & 198.8 & $57.5 \%$ & 7.1 & $23.8 \%$ & 5.2 & $0.0 \%$ & 7.6 \\
\hline $25-3$ & 9224.7 & 216.2 & $42.8 \%$ & 7.1 & $6.8 \%$ & 5.3 & $0.0 \%$ & 7.1 \\
\hline $25-4$ & 6637.8 & 231.8 & $1.5 \%$ & 8.0 & $60.5 \%$ & 6.0 & $0.0 \%$ & 8.4 \\
\hline $36-1$ & 10681.7 & 1673.3 & $37.4 \%$ & 18.1 & $59.6 \%$ & 13.0 & $0.0 \%$ & 18.6 \\
\hline $36-2$ & 71703.2 & 3629.1 & $39.3 \%$ & 36.9 & $28.9 \%$ & 27.6 & $0.0 \%$ & 39.4 \\
\hline $36-3$ & 55237.9 & 2997.7 & $0.0 \%$ & 31.8 & $67.8 \%$ & 22.3 & $0.0 \%$ & 32.4 \\
\hline $36-4$ & 9284.0 & 1598.5 & $70.5 \%$ & 17.4 & $63.4 \%$ & 12.0 & $0.0 \%$ & 18.0 \\
\hline $16-1 \mathrm{~h}$ & 61193.4 & 31.6 & $0.0 \%$ & 5.8 & $0.0 \%$ & 3.9 & $0.0 \%$ & 5.4 \\
\hline $16-2 \mathrm{~h}$ & 117441.0 & 38.6 & $23.6 \%$ & 5.5 & $38.1 \%$ & 4.6 & $0.0 \%$ & 6.8 \\
\hline $16-3 \mathrm{~h}$ & 368930.0 & 34.3 & $0.0 \%$ & 7.2 & $6.0 \%$ & 4.2 & $0.0 \%$ & 6.0 \\
\hline $16-4 \mathrm{~h}$ & 96677.3 & 41.1 & $0.9 \%$ & 8.0 & $0.0 \%$ & 4.9 & $0.0 \%$ & 7.1 \\
\hline $25-1 \mathrm{~h}$ & 111784.0 & 423.7 & $25.9 \%$ & 16.2 & $26.2 \%$ & 12.4 & $0.0 \%$ & 17.3 \\
\hline $25-2 \mathrm{~h}$ & 119014.0 & 405.8 & $4.5 \%$ & 14.3 & $4.5 \%$ & 10.9 & $0.0 \%$ & 15.8 \\
\hline $25-3 \mathrm{~h}$ & 76977.7 & 441.3 & $11.5 \%$ & 17.4 & $11.9 \%$ & 13.1 & $0.0 \%$ & 19.8 \\
\hline $25-4 \mathrm{~h}$ & 114635.0 & 398.6 & $12.7 \%$ & 15.8 & $0.0 \%$ & 10.7 & $0.0 \%$ & 15.2 \\
\hline $36-1 \mathrm{~h}$ & 172687.0 & 4436.6 & $65.5 \%$ & 44.6 & $0.0 \%$ & 36.1 & $0.0 \%$ & 54.6 \\
\hline $36-2 \mathrm{~h}$ & 218958.0 & 3625.7 & $70.3 \%$ & 42.0 & $78.3 \%$ & 30.5 & $0.0 \%$ & 48.1 \\
\hline $36-3 \mathrm{~h}$ & 124627.0 & 4231.3 & $82.9 \%$ & 45.9 & $0.0 \%$ & 33.8 & $0.0 \%$ & 49.4 \\
\hline $36-4 \mathrm{~h}$ & 80600.3 & 3360.3 & $38.1 \%$ & 39.2 & $0.9 \%$ & 27.9 & $0.0 \%$ & 43.5 \\
\hline Sioux & 6539.2 & 143.0 & $0.0 \%$ & 5.2 & $0.0 \%$ & 3.9 & $0.0 \%$ & 6.0 \\
\hline Sioux-h & 29.7 & 21.0 & $0.0 \%$ & 25.4 & $0.0 \%$ & 12.1 & $0.0 \%$ & 18.2 \\
\hline Average & & 1093.7 & $28.0 \%$ & 16.9 & $19.3 \%$ & 12.1 & $0.0 \%$ & 17.9 \\
\hline Median & & 224.0 & $23.2 \%$ & 11.1 & $6.4 \%$ & 8.4 & $0.0 \%$ & 11.8 \\
\hline IQR & & 1970.8 & $52.6 \%$ & 21.3 & $31.2 \%$ & 11.3 & $0.0 \%$ & 16.9 \\
\hline Min & & 13.7 & $0.0 \%$ & 2.6 & $0.0 \%$ & 1.8 & $0.0 \%$ & 2.4 \\
\hline $\operatorname{Max}$ & & 4436.6 & $82.9 \%$ & 45.9 & $78.3 .0 \%$ & 36.1 & $0.0 \%$ & 54.6 \\
\hline
\end{tabular}


Table 5. Comparison of solution approaches when 2 arcs are disrupted $(R=4)$.

\begin{tabular}{|c|c|c|c|c|c|c|c|c|}
\hline \multirow[b]{2}{*}{$\begin{array}{l}\text { Instance } \\
\text { Name }\end{array}$} & \multicolumn{2}{|c|}{ Enumeration } & \multicolumn{2}{|c|}{$\mathrm{RG}$} & \multicolumn{2}{|c|}{ MSG } & \multicolumn{2}{|c|}{ MSG-SAS } \\
\hline & Obj & $\begin{array}{l}\text { CPU } \\
\text { Time(s) }\end{array}$ & $\operatorname{Gap}(\%)$ & $\begin{array}{l}\text { CPU } \\
\text { Time(s) }\end{array}$ & $\operatorname{Gap}(\%)$ & $\begin{array}{l}\text { CPU } \\
\text { Time(s) }\end{array}$ & $\operatorname{Gap}(\%)$ & $\begin{array}{l}\text { CPU } \\
\text { Time(s) }\end{array}$ \\
\hline $16-1$ & 27599.9 & 205.8 & $0.0 \%$ & 32.4 & $0.0 \%$ & 28.8 & $0.0 \%$ & 29.8 \\
\hline $16-2$ & 72826.3 & 230.1 & $0.0 \%$ & 42.2 & $0.0 \%$ & 33.6 & $0.0 \%$ & 34.7 \\
\hline $16-3$ & 42836.1 & 235.4 & $11.4 \%$ & 34.4 & $8.0 \%$ & 35.2 & $0.0 \%$ & 36.2 \\
\hline $16-4$ & 19864.6 & 166.6 & $0.0 \%$ & 27.1 & $0.0 \%$ & 22.9 & $0.0 \%$ & 23.7 \\
\hline $25-1$ & 66175.7 & 3778.4 & $0.0 \%$ & 108.4 & $0.0 \%$ & 75.0 & $0.0 \%$ & 78.4 \\
\hline $25-2$ & 53918.3 & 3987.7 & $3.1 \%$ & 80.2 & $0.0 \%$ & 69.6 & $0.0 \%$ & 72.3 \\
\hline $25-3$ & 23207.9 & 4138.3 & $0.0 \%$ & 71.4 & $0.0 \%$ & 69.0 & $0.0 \%$ & 71.1 \\
\hline $25-4$ & 29670.0 & 4482.2 & $31.0 \%$ & 95.2 & $0.0 \%$ & 78.6 & $0.0 \%$ & 81.5 \\
\hline $36-1$ & & & $0.0^{\dagger} \%$ & 203.0 & $32.9^{\dagger} \%$ & 190.6 & $1.9 \%^{\dagger}$ & 198.7 \\
\hline $36-2$ & & & $23.6^{\dagger} \%$ & 393.4 & $3.2^{\dagger} \%$ & 392.5 & $0.0 \%^{\dagger}$ & 405.4 \\
\hline $36-3$ & & & $38.5^{\dagger} \%$ & 332.4 & $0.0^{\dagger} \%$ & 319.5 & $0.0 \%^{\dagger}$ & 331.0 \\
\hline $36-4$ & & & $0.0^{\dagger} \%$ & 239.0 & $0.4^{\dagger} \%$ & 169.2 & $0.0 \%^{\dagger}$ & 177.7 \\
\hline $16-1 \mathrm{~h}$ & 235197.0 & 393.5 & $24.5 \%$ & 53.9 & $0.0 \%$ & 53.1 & $0.0 \%$ & 54.5 \\
\hline $16-2 \mathrm{~h}$ & 340356.0 & 471.1 & $14.9 \%$ & 57.7 & $0.0 \%$ & 65.6 & $0.0 \%$ & 67.6 \\
\hline $16-3 \mathrm{~h}$ & 1259220.0 & 406.7 & $0.0 \%$ & 70.8 & $0.0 \%$ & 58.0 & $0.0 \%$ & 59.6 \\
\hline $16-4 \mathrm{~h}$ & 482228.0 & 479.5 & $0.0 \%$ & 66.9 & $0.0 \%$ & 65.2 & $0.0 \%$ & 66.9 \\
\hline $25-1 \mathrm{~h}$ & 349404.0 & 8945.5 & $31.2 \%$ & 139.1 & $0.0 \%$ & 171.1 & $0.0 \%$ & 176.2 \\
\hline $25-2 \mathrm{~h}$ & 274589.0 & 8377.1 & $0.0 \%$ & 141.7 & $3.0 \%$ & 155.6 & $0.0 \%$ & 160.1 \\
\hline $25-3 \mathrm{~h}$ & 557922.0 & 9293.6 & $0.0 \%$ & 182.7 & $0.0 \%$ & 201.3 & $0.0 \%$ & 208.4 \\
\hline $25-4 h$ & 646824.0 & 8283.2 & $58.7 \%$ & 143.5 & $0.0 \%$ & 159.5 & $0.0 \%$ & 164.2 \\
\hline $36-1 \mathrm{~h}$ & & & $18.0 \% \dagger$ & 460.3 & $0.0 \%^{\dagger}$ & 513.6 & $0.0 \%^{\dagger}$ & 528.5 \\
\hline $36-2 \mathrm{~h}$ & & & $66.8 \%^{\dagger}$ & 504.7 & $66.8 \%^{\dagger}$ & 440.0 & $0.0 \%^{\dagger}$ & 455.8 \\
\hline $36-3 \mathrm{~h}$ & & & $10.1 \%^{\dagger}$ & 542.7 & $9.5 \%^{\dagger}$ & 464.1 & $0.0 \%^{\dagger}$ & 478.4 \\
\hline $36-4 h$ & & & $6.4 \%^{\dagger}$ & 470.1 & $0.0 \%{ }^{\dagger}$ & 393.5 & $0.0 \%^{\dagger}$ & 410.0 \\
\hline Sioux & 82376.3 & 2892.9 & $0.0 \%$ & 59.0 & $1.3 \%$ & 61.9 & $0.0 \%$ & 65.1 \\
\hline Sioux-h & 2576870.0 & 11439.9 & $0.0 \%$ & 249.4 & $0.0 \%$ & 259.6 & $0.0 \%$ & 268.8 \\
\hline Average & & 3789.3 & $13.0 \%$ & 184.7 & $4.8 \%$ & 174.9 & $0.1 \%$ & 180.9 \\
\hline Median & & 3335.7 & $1.5 \%$ & 123.8 & $0.0 \%$ & 117.1 & $0.0 \%$ & 120.8 \\
\hline IQR & & 7952.7 & $23.8 \%$ & 211.5 & $1.7 \%$ & 213.6 & $0.0 \%$ & 220.7 \\
\hline Min & & 166.6 & $0.0 \%$ & 27.1 & $0.0 \%$ & 22.9 & $0.0 \%$ & 23.7 \\
\hline Max & & 11439.9 & $66.8 \%$ & 542.7 & $66.8 \%$ & 513.6 & $1.9 \%$ & 528.5 \\
\hline
\end{tabular}

${ }^{\dagger}$ Percentage gap from the best solution found. 
on the grids for which optimal gaps can be computed, while changing the seed for the pseudo-random function. The analysis is repeated 50 times and the objective percentage gaps between MSG-SAS and Enumeration (averaged across all network instances) are shown in Figure 2. The $R=4$ case is excluded since no gaps can be computed for the 36-node instances.

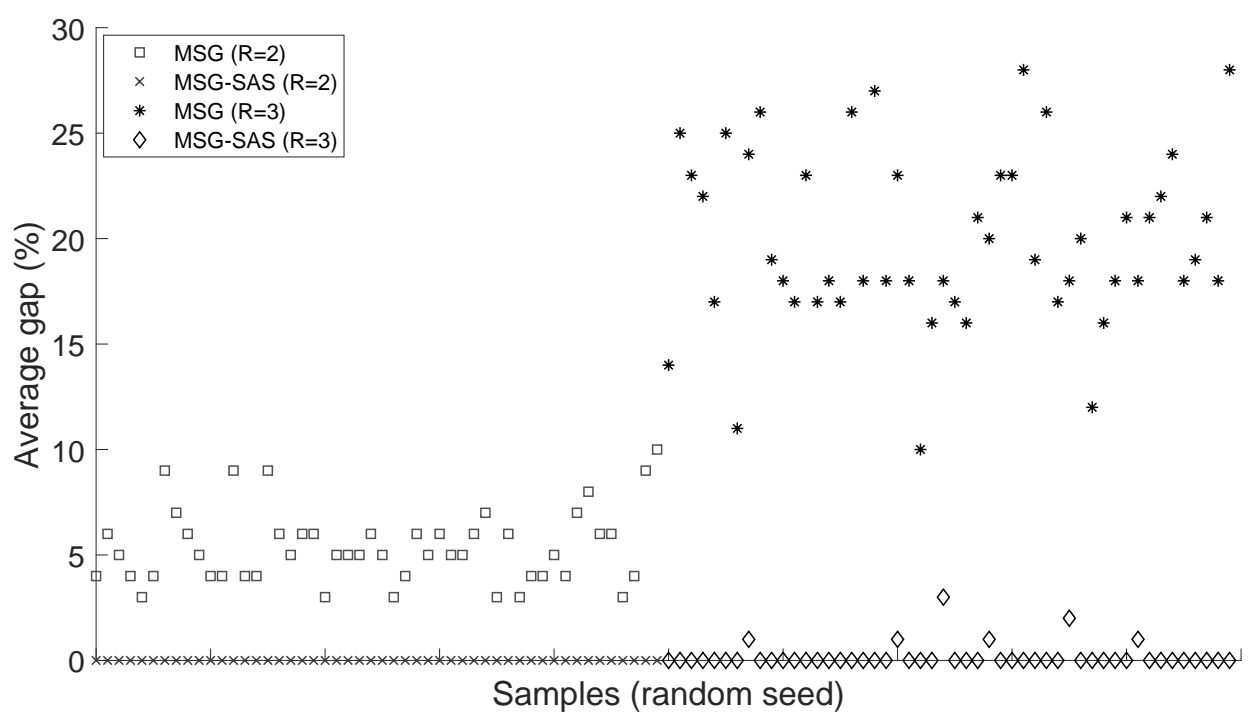

Figure 2. Average gap (\%) for MSG and MSG-SAS when $R=2$ and $R=3$, varying the random seeds.

The graphs highlights that MSG results are variable. When $R=2$, its gaps range between $\sim 3 \%$ and $\sim 10 \%$ with a standard deviation of $1.7 \%$. This variability further increases when $R=3$, with the gaps often approaching $30 \%$, while never being smaller than $10 \%$ and the standard deviation rising to $4.2 \%$. It is clear that adding the search step after MSG has the benefits of bringing the gaps to negligible levels while also reducing the aforementioned variability (standard deviation reduces to $0.1 \%$ and $0.6 \%$, respectively). A few sporadic outliers appear with MSG-SAS and $\mathrm{R}=3$.Among these, only two result in a gap greater than $1 \%$.

The scalability of the algorithm is tested on larger grids and Berlin network. For brevity, we report only the results when $R=4$, since it is the most computationally expensive in our analysis. Across all instances, MSG-SAS returns solutions far more accurate than RG. The gap between the two procedures ranges between $54 \%$ and $328 \%$.

Table 6. Computational results for larger networks $(R=4)$.

\begin{tabular}{llll}
\hline & RG & \multicolumn{2}{c}{ MSG-SAS } \\
\cline { 3 - 4 } Instance & CPU & CPU & Gap from \\
Name & Time(s) & Time(s) & RG(\%) \\
\hline 64 & 868 & 809 & $57 \%$ \\
$64 \mathrm{~h}$ & 1,520 & 1,464 & $94 \%$ \\
100 & 2,600 & 2,366 & $328 \%$ \\
$100 \mathrm{~h}$ & 5,925 & 5,644 & $54 \%$ \\
Berlin & 5,869 & 5,673 & $211 \%$ \\
\hline
\end{tabular}




\section{Case Study}

In this section we use the Sioux network to provide some managerial insights. The network is again evaluated in congestion and high-congestion conditions.

\subsection{Solutions Analysis}

Figures 3 shows a graphical representation of the consequences of disruptions on the traffic congestion. Arcs are drawn correlating thickness with congestion levels. Furthermore, disrupted arcs are represented by dotted lines.

Figures $3 \mathrm{a}$ and $3 \mathrm{~b}$ show the networks after a worst-case loss of $R=2$ arcs. Similarly, Figures $3 \mathrm{c}$ and $3 \mathrm{~d}$, show the post-disruption networks when $R=4$. These results suggest that the worst critical loss of 2 arcs (namely, 43 and 60) generates congestions around nodes 17 and 19. When $R=4,43$ and 60 are still the most critical arcs, along with 38 and 58. Their loss generates a vast congested area around nodes 11, 14, 15 and 23. This congestion is particularly severe for the high congestion case, as shown by figure 3d. Overall, the figures suggest that arcs with high traffic tend to be also the most critical. For example, arc 58 (from node 17 to 19) is the most congested after the worst case loss of two arcs and consequently appears among the most critical arcs when $R=4$.

\subsection{Comparison with Vulnerability Metrics}

This analysis already suggests that when assessing vulnerabilities an important factor is played by the correlation between arcs. To further explore this issue, we compare the results of UEIM against some popular vulnerability metrics. One of the most frequently used approach is to remove arcs (and nodes) one by one from the network and measure the system's performances before and after. Typically these performances are assessed in terms of travel times or costs. The first metric considered is the importance of an arc and or node (IMP) introduced by Jenelius et al. (2006). It is formally defined as follows:

$$
\operatorname{IMP}(a)=\frac{\sum_{r} \sum_{s \neq r} q_{r s}\left(c_{r s}^{a}-c_{r s}^{0}\right)}{\sum_{r} \sum_{s \neq r} q_{r s}}
$$

where $c_{r s}^{0}$ and $c_{r s}^{a}$ are respectively the cost of travel from $r$ to $s$ with and without arc $a$. In our analysis these costs are computed as the shortest travel time, assuming free flow on each arc. In other words, the metrics does not account for congestion.

The second metrics being evaluated is introduced by Scott et al. (2006), referred to as Network Robustness Index (NRI). This measure is defined as follows:

$$
N R I(a)=\sum_{a} x_{a} t\left(x_{a}\right) \delta_{a}
$$

with $\delta_{a}$ equal to 1 if arc $a$ is operational, 0 otherwise. In other words, the metric evaluates arc $a$ by removing it from the network, finding the new equilibrium and assessing the cost increment. 

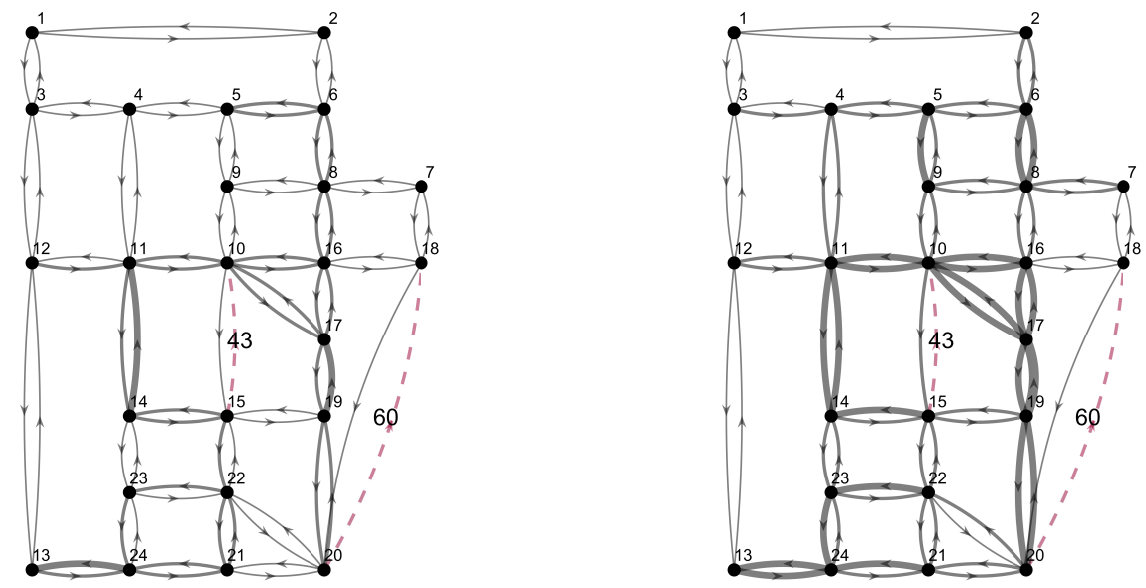

(a) Congested, with $R=2$.

(b) Heavily congested, with $R=2$.
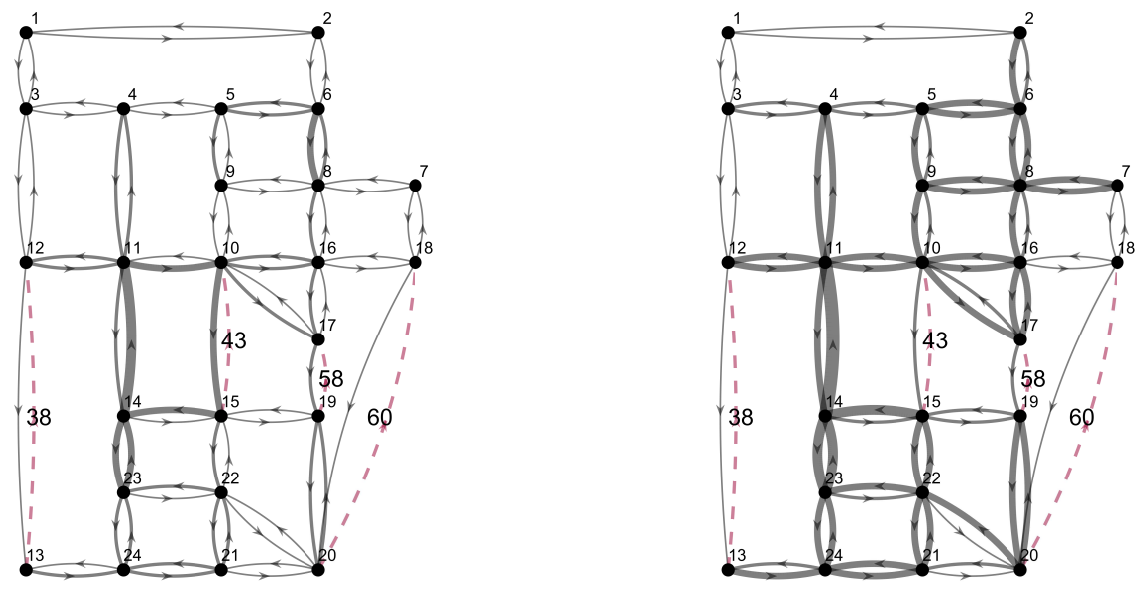

(c) Congested, with $R=4$.

(d) Heavily congested, with $R=4$.

Figure 3. Interdiction plans and congestion on the Sioux network 
Table 7 compares the rankings of IMP and NRI with the solutions of UEIM, when Sioux is heavily congested. The first three columns show rank and arc index for IMP and NRI, respectively. The last column shows the UEIM solution obtained with the $\mathrm{R}$ value reported in the fourth column. The table clearly highlights differences between the metrics and the UEIM's solutions. In fact, when $R$ is 2 the optimisation approach suggests that arc 60 (together with 43) is the most critical asset. This is in sharp contrast with both IMP and NRI which rank it 64th and 69th (out of 76 arcs), respectively.

Table 7. Critical arcs according to IMP, NRI and UEIM

\begin{tabular}{|c|c|c|c|c|}
\hline \multirow[b]{2}{*}{ Rank } & IMP & NRI & \multicolumn{2}{|r|}{ UEIM } \\
\hline & Arc & Arc & $\mathrm{R}$ & Arcs \\
\hline 1 & 26 & 43 & 1 & 43 \\
\hline 2 & 25 & 28 & 2 & 4360 \\
\hline 3 & 16 & 37 & 3 & 435860 \\
\hline 4 & 19 & 38 & 4 & 38435860 \\
\hline 5 & 49 & 26 & 5 & 3840435260 \\
\hline 6 & 52 & 25 & 6 & $\begin{array}{llllll}38 & 40 & 43 & 52 & 53 & 60\end{array}$ \\
\hline 7 & 27 & 46 & 7 & 9162532435860 \\
\hline 8 & 48 & 67 & 8 & 711192627285356 \\
\hline
\end{tabular}

The difference between rankings and UEIM's solutions is also evident when taking into account the system's costs. In the following analysis, we compute the total system's cost by sequentially disrupting an increasing number $R$ of arcs, according to the priority order identified by IMP and NRI rankings. Results are then compared with the system's costs generated by UEIM (assuming the same value of $R$ ).

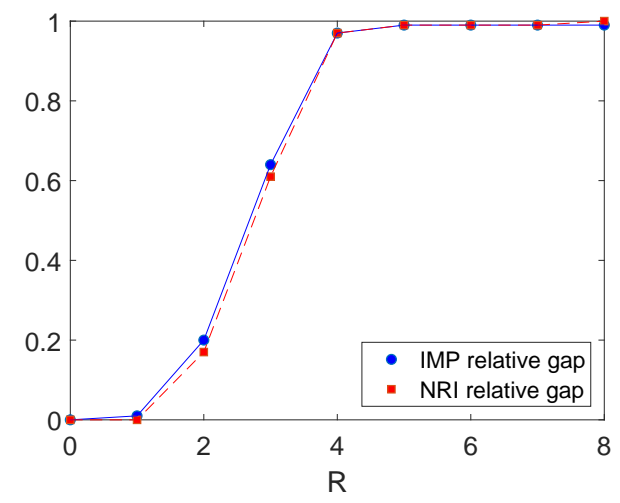

(a) Congested.

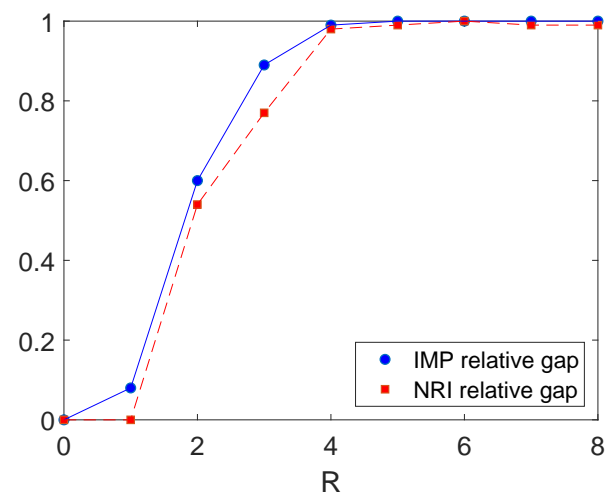

(b) Heavily congested.

Figure 4. IMP, NRI versus UEIM. Relative error.

Results of this analysis are reported in Figure 4 displaying the relative gap between metrics' and UEIM's objectives. Both congestion cases are evaluated. The graphs show that the performances of these metrics quickly degrade as more disruptions are considered. In fact, already for $R$ equal to 2, IMP and NRI heavily underestimate the vulnerability. For $R$ greater than 4 , the metrics return solutions smaller than UEIM's by one or even two orders of magnitude. The two graphs show differences only for smaller values of $R$. For example, when $R=2,3$, the gaps obtained on the congested network are smaller compared with the counterparts obtained on the heavily congestion network (e.g., IMP gaps increase from about $20 \%$ to $60 \%$, when $R=2$ ). 
Overall, UEIM is a better way to assess network vulnerability to multiple failures as it captures the interdependence among different components. The simultaneous loss of several arcs can be much more severe than what the metrics suggest.

To see if similar results can be drawn on networks of different sizes and structures, we perform the same comparison on the set of large networks considered section 4 . The results (Figures 5a, 5b) are indeed consistent with those observed on the Sioux case study. In fact, as $R$ increases, the gaps between NRI, IMP and UEIM approach $100 \%$ with small differences across networks.

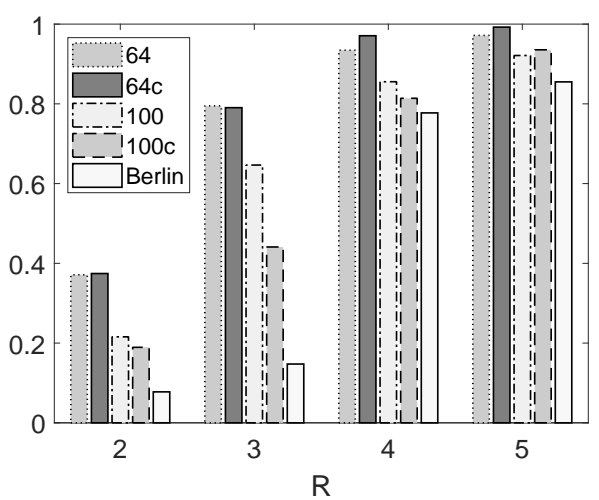

(a) IMP.

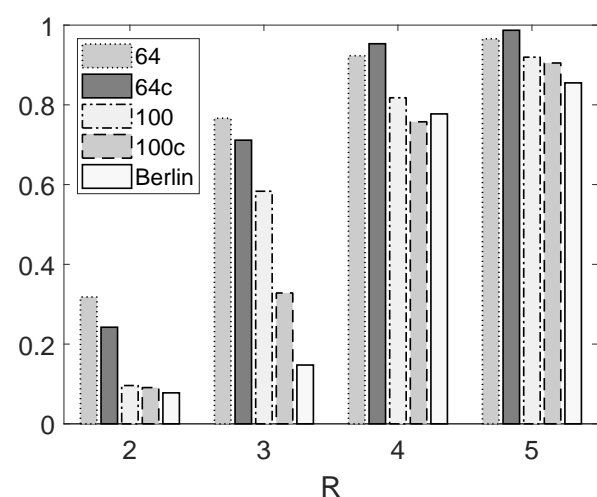

(b) NRI.

Figure 5. IMP, NRI versus UEIM. Relative error on large networks.

\subsection{Protecting the Road Network}

This section discusses how UEIM can be used to support actions to decrease system's vulnerability. We assume that a limited number of arcs can be fortified and made invulnerable to disruptions. For example, if the road infrastructure is vulnerable to flooding, temporary and permanent barriers can be built along arcs to allow them to withstand higher flooding levels. A thorough approach would require to explicitly model this decision process. Formally, a third optimisation level (often referred to as fortification level) is needed in the UEIM mathematical formulation to model the decision of which arcs should be fortified, given a limited available budget. Examples of fortification papers focusing on supply-chain, shortest-path and flow-based networks can be found in Cappanera and Scaparra (2011); Church and Scaparra (2007); Starita and Scaparra $(2016,2018)$. Unfortunately, this added optimisation level further complicates an already challenging problem and a new solution approach would have to be devised. Being aware of this and leaving this extension as a future development, here we evaluate the benefits of using UEIM results to decide which arc to protect. We compare three different decision making policies. Let us refer to $Q$ as the number of arcs to protect, $I S$ as the set of most critical arcs generated by MSG-SAS and FS as the set of fortified arcs defined by one of the following policies. The first policy, referred to as NRI-P, is based on the ranking according to NRI metric. For example, for the high congestion case when $Q=3, F S=\{43,28,37\}$. The second policy, referred to as UEIM-P1, initialise $F S$ to the $I S$ obtained when $R=Q$. Finally, the following pseudo-code describes the third policy, referred to as UEIM-P2.

UEIM-P2 iteratively selects which arcs to fortify by randomly choosing from the set of most critical arcs generated by UEIM, assuming that arcs already in FS cannot 


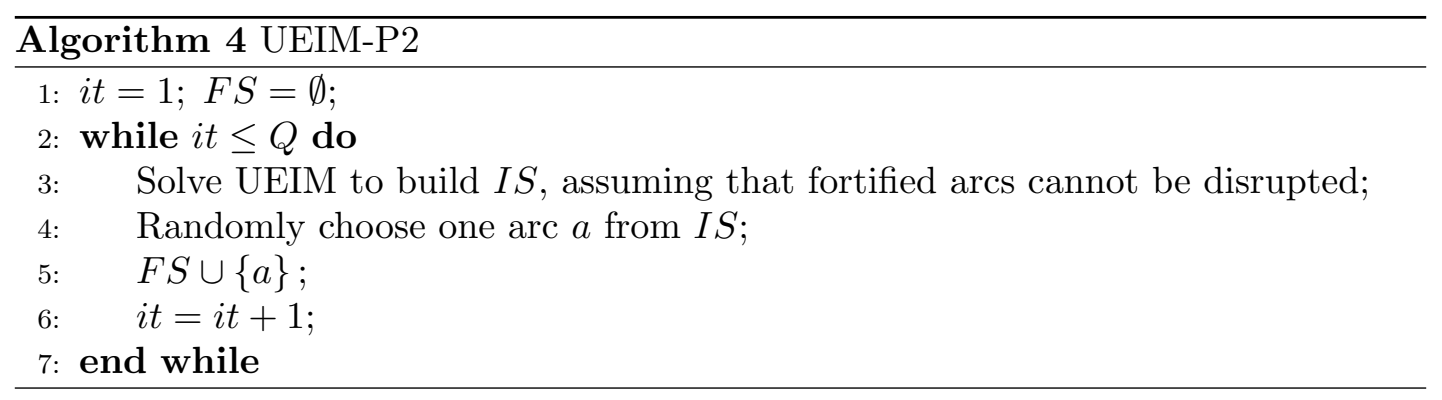

be disrupted.

We test these policies by evaluating what is the worst case cost after loosing the 3 most critical unprotected arcs. Figures $6 \mathrm{a}$ and $6 \mathrm{~b}$ show the percentage decrease of the worst-case system cost (for both congestion cases) when an increasing number of arcs are protected according to the different policies.

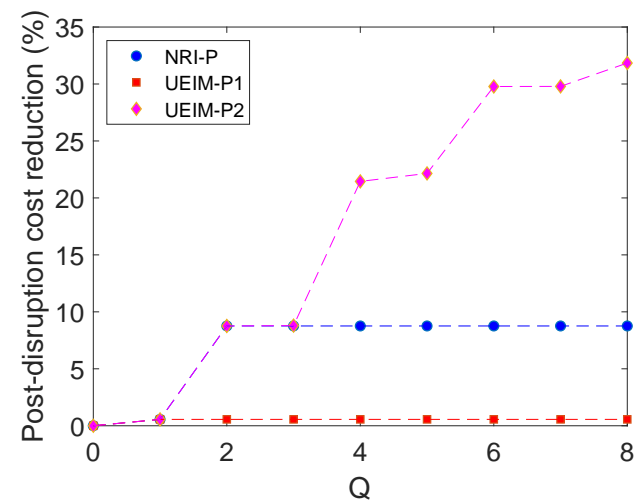

(a) Congested

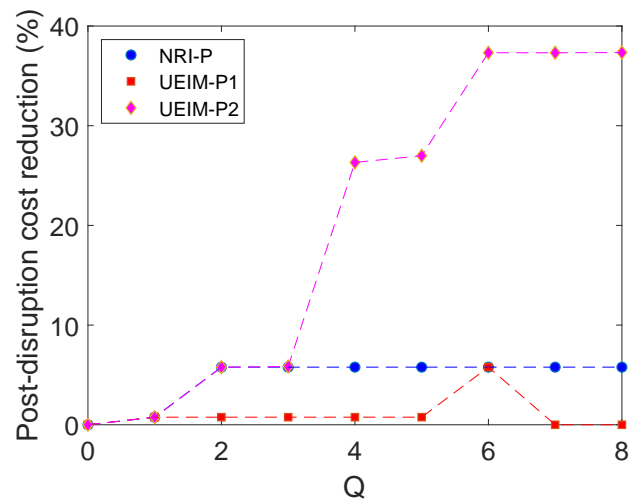

(b) Heavily congested

Figure 6. Comparison among fortification policies.

In general, the two congestion cases do not show major differences. In both, UEIM$\mathrm{P} 1$ performs poorly, failing to reduce the post-disruption system's cost. This is because its fortification sets are based on worst case disruptions computed assuming an unprotected network and therefore fails to adapt to new worst case solutions discovered as arcs are being fortified. In fact, after successfully thwarting the worst-case disruption when $Q=1$, UEIM-P1 almost always fails to improve because the new worst-case sets and the fortification sets have no arcs in common. In other words, UEIM-P1 too often fortifies arcs that do not need fortification. A similar issue is faced by NRI-P, although this policy manages to achieve a slightly higher cost decrement (about $8.8 \%$ and $5.8 \%$ for congested and heavily congested, respectively). Overall, the graphs suggest that both NRI-P and UEIM-P1 are not suitable policies to support fortification decisions. Conversely, UEIM-P2 shows rather promising results. The system's cost is gradually reduced in both cases, as $Q$ increases. The reason is that UEIM-P2 better tackles the game between fortification and interdiction, by sequentially choosing arcs from newly found interdiction sets. When $Q=8$, UEIM-P2 reduces the post-disruption system's cost by $31.8 \%$ in the congested case. Not surprisingly, with high congestion the peak reduction is even higher $(37.3 \%)$ and achieved with fewer fortifications $(Q=6)$. This can be explained with the potentially larger room for improvement to be expected in 
a highly congested environment.

The advantage of UEIM-P2 against the NRI-P and UEIM-P1 is further confirmed when larger grids and the Berlin network are considered. Table 8 summarises the post-disruption cost reduction obtained by all policies when $Q=1,4$ and 8 . With the only exception of grid 100 when $Q=1$, UEIM-P2 always performs better. As seen on Sioux network, the extent of the benefit of using UEIM-P2 increases with the protection budget achieving cost reductions ranging from $15 \%$ to $65 \%$.

Table 8. Post-disruption cost reduction(\%) on large networks when $R=3$

\begin{tabular}{lllllll}
\hline & & \multicolumn{5}{c}{ Instance Name } \\
\cline { 2 - 7 } $\mathrm{Q}$ & Policy & 64 & $64 \mathrm{~h}$ & 100 & $100 \mathrm{~h}$ & Berlin \\
\hline \multirow{3}{*}{1} & NRI-P & $\mathbf{0 \%}$ & $0 \%$ & $0 \%$ & $0 \%$ & $0 \%$ \\
& UEIM-P1 & $\mathbf{0 \%}$ & $0 \%$ & $\mathbf{6 4 \%}$ & $0 \%$ & $0 \%$ \\
& UEIM-P2 & $\mathbf{0 \%}$ & $\mathbf{2 9 \%}$ & $4 \%$ & $\mathbf{3 \%}$ & $\mathbf{8 \%}$ \\
\multirow{3}{*}{4} & NRI-P & $0 \%$ & $0 \%$ & $24 \%$ & $0 \%$ & $0 \%$ \\
& UEIM-P1 & $27 \%$ & $0 \%$ & $53 \%$ & $0 \%$ & $0 \%$ \\
& UEIM-P2 & $\mathbf{4 4 \%}$ & $\mathbf{3 8 \%}$ & $\mathbf{5 4 \%}$ & $\mathbf{1 3 \%}$ & $\mathbf{1 4 \%}$ \\
& NRI-P & $27 \%$ & $0 \%$ & $57 \%$ & $13 \%$ & $0 \%$ \\
& UEIM-P1 & $0 \%$ & $0 \%$ & $53 \%$ & $0 \%$ & $8 \%$ \\
& UEIM-P2 & $\mathbf{6 5 \%}$ & $\mathbf{5 6 \%}$ & $\mathbf{5 8 \%}$ & $\mathbf{1 5 \%}$ & $\mathbf{1 7 \%}$ \\
\hline
\end{tabular}

\section{Concluding remarks}

In this paper, we propose an optimisation approach to assess road network vulnerability. We introduce an interdiction model on an User Equilibrium network to estimate the impact of worst-case disruptions on the congestion of a system. The bi-level nonlinear formulation is challenging to solve. Therefore, a heuristic inspired by GRASP is developed. Computational results performed on artificial grids show that the heuristic is fast and accurate. Furthermore, experiments on two real case studies, (Sioux Fall and Berlin Friedrichshain) clearly show the benefit of incorporating results from our model into the decisional process of planning for road network protections and enhancements. Overall, the experiments prove that if multiple disruptions are considered, optimisation approaches are superior to approaches based on metrics. This is because once an arc fails, the criticality of the other arcs changes. Static approaches based on rankings cannot capture this issue.

Several lines of research can be pursued to further expand this paper. The first natural extension will be to introduce a fortification level to explicitly include protection investment decisions. Focusing on natural hazards, it makes sense to introduce geographically correlated disruption, as done by Liberatore et al. (2012).

One ambitious extension is assessing vulnerability within a multi-modal transportation context, where disruption over one infrastructure have a ripple effect on the others. In general, the temporal component has been neglected in interdiction and fortification models. For example, time can be included in the formulation in order to study interesting cases such as sequential disruptions, protection with lifespans, interdiction and disruption budget available over a time horizon, long-lasting impact of disruptions etc. Another realistic extension would be to consider interdictions causing partial damages (e.g., partial closure of a road, closure of some lanes etc.). These assumptions have already been considered in interdiction models applied to supply chain problems (Aksen et al., 2014; Zhang et al., 2016). 
Finally, these suggested extensions are bound to complicate the mathematical formulation of the problem even further. Therefore, future developments within this research area should include the exploration of new efficient solution procedures.

\section{References}

Afshari Rad, M. and Kakhki, H. T. (2013). Maximum dynamic network flow interdiction problem: New formulation and solution procedures. Computers 83 Industrial Engineering, 65(4):531-536.

Akbari-Jafarabadi, M., Tavakkoli-Moghaddam, R., Mahmoodjanloo, M., and Rahimi, Y. (2017). A tri-level r-interdiction median model for a facility location problem under imminent attack. Computers 83 Industrial Engineering, 114:151-165.

Aksen, D., Akca, S. Ş., and Aras, N. (2014). A bilevel partial interdiction problem with capacitated facilities and demand outsourcing. Computers $\mathcal{E}$ Operations Research, 41:346358.

Aksen, D., Aras, N., and Piyade, N. (2013). A Bilevel p-median model for the planning and protection of critical facilities. Journal of Heuristics, 19(2):373-398.

Bekhor, S. and Toledo, T. (2005). Investigating path-based solution algorithms to the stochastic user equilibrium problem. Transportation Research Part B: Methodological, 39(3):279295.

Bidgoli, M. M. and Kheirkhah, A. (2018). An arc interdiction vehicle routing problem with information asymmetry. Computers \& Industrial Engineering, 115:520-531.

Blumenfeld, H. (1949). Theory of city form, past and present. Journal of the society of architectural historians, 8(3/4):7-16.

Brown, G., Carlyle, M., Salmerón, J., and Wood, K. (2006). Defending Critical Infrastructure. Interfaces, 36(6):530-544.

Bruynooghe, M. (1972). An optimal method of choice of investments in a transport network. PTRC Proceedings.

Bureau of Transportation Statistics (2017a). 2016 North American Freight Numbers. Avaliable at: https://www.bts.gov/newsroom/2016-north-american-freight-numbers Last time accessed: 2018-08-10.

Bureau of Transportation Statistics (2017b). Transportation Statistics Annual Report . Avaliable at: http://www.transtats.bts.gov. Last time accessed: 2018-08-10. Technical report.

Cadarso, L. and Marín, Á. (2016). Combining robustness and recovery in rapid transit network design. 12(3):203-229.

Cappanera, P. and Scaparra, M. P. (2011). Optimal Allocation of Protective Resources in Shortest-Path Networks. Transportation Science, 45(1):64-80.

Chen, L. and Miller-Hooks, E. (2012). Resilience: An indicator of recovery capability in intermodal freight transport. Transportation Science, 46(1):109-123.

Chiou, S.-W. (2005). Bilevel programming for the continuous transport network design problem. Transportation Research Part B: Methodological, 39(4):361-383.

Church, R. L. and Scaparra, M. P. (2007). Protecting Critical Assets: The r-Interdiction Median Problem with Fortification. Geographical Analysis, 39(2):129-146.

Church, R. L., Scaparra, M. P., and Middleton, R. S. (2004). Identifying Critical Infrastructure: The Median and Covering Facility Interdiction Problems. Annals of the Association of American Geographers, 94(3):491-502.

Department for Transport (2016). Transport Statistics Great Britain 2016. Available at: https://assets.publishing.service.gov.uk Last time accessed: 2018-08-10. Technical report.

Dimitriou, L., Stathopoulos, A., and Tsekeris, T. (2008). Reliable stochastic design of road network systems. International Journal of Industrial and Systems Engineering, 3(5):549574 .

Drezner, Z. and Wesolowsky, G. O. (2003). Network design: selection and design of links and 
facility location. Transportation Research Part A: Policy and Practice, 37(3):241-256.

El-Rashidy, R. A. and Grant-Muller, S. M. (2014). An assessment method for highway network vulnerability. Journal of Transport Geography, 34:34-43.

Environmental Agency (2018). Estimating the economic costs of the 2015 to 2016 winter floods. Avaliable at: www.gov.uk/environment-agency Last time accessed: 2018-08-10. Technical report.

Faturechi, R. and Miller-Hooks, E. (2014). Travel time resilience of roadway networks under disaster. Transportation Research Part B: Methodological, 70:47-64.

Feo, T. A. and Resende, M. G. C. (1995). Greedy Randomized Adaptive Search Procedures. Journal of Global Optimization, 6(2):109-133.

Fulkerson, D. R. and Harding, G. C. (1977). Maximizing the minimum source-sink path subject to a budget constraint. Mathematical Programming, 13(1):116-118.

Golany, B., Kaplan, E. H., Marmur, A., and Rothblum, U. G. (2009). Nature plays with dice-terrorists do not: Allocating resources to counter strategic versus probabilistic risks. European Journal of Operational Research, 192(1):198-208.

He, X. and Liu, H. X. (2012). Modeling the day-to-day traffic evolution process after an unexpected network disruption. Transportation Research Part B: Methodological, 46(1):5071.

Ishfaq, R. (2013). Intermodal shipments as recourse in logistics disruptions. Journal of the Operational Research Society, 64(2):229-240.

Israeli, E. and Wood, R. K. (2002). Shortest-path network interdiction. Networks, 40(2):97111.

Jahn, O., Möhring, R. H., Schulz, A. S., and Stier-Moses, N. E. (2005). System-optimal routing of traffic flows with user constraints in networks with congestion. Operations research, $53(4): 600-616$.

Jenelius, E. and Mattsson, L. G. (2012). Road network vulnerability analysis of area-covering disruptions: A grid-based approach with case study. Transportation Research Part A: Policy and Practice, 46(5):746-760.

Jenelius, E., Petersen, T., and Mattsson, L. G. (2006). Importance and exposure in road network vulnerability analysis. Transportation Research Part A: Policy and Practice, 40(7):537560.

Jin, L., Wang, H., Xie, B., Yu, L., and Liu, L. (2017). A user exposure based approach for non-structural road network vulnerability analysis. PLOS ONE, 12(11):e0188790.

Kelly J., C., David P., M., and R. Kevin, W. (1998). Stochastic Network Interdiction. Operations Research, 46(2):184-197.

Kepaptsoglou, K. L., Konstantinidou, M. A., Karlaftis, M. G., and Stathopoulos, A. (2014). Planning postdisaster operations in a highway network: network design model with interdependencies. Transportation Research Record, 2459(1):1-10.

Kermanshah, A. and Derrible, S. (2017). Robustness of road systems to extreme flooding: using elements of gis, travel demand, and network science. Natural Hazards, 86(1):151-164.

Kim, B. J. and Kim, W. (2006). An equilibrium network design model with a social cost function for multimodal networks. The Annals of Regional Science, 40(3):473-491.

Lansing State Journal (2019). Multiple roads in eaton county closed due to flooding. (Accessed on $08 / 06 / 2019)$.

Laporte, G., Marín, A., Mesa, J. A., and Perea, F. (2011). Designing robust rapid transit networks with alternative routes. Journal of Advanced Transportation, 45(1):54-65.

Leblanc, L. J. (1975). An Algorithm for the Discrete Network Design Problem. Transportation Science, 9(3):183-199.

Leng, J.-q., Zhai, J., Li, Q.-w., and Zhao, L. (2018). Construction of road network vulnerability evaluation index based on general travel cost. Physica A: Statistical Mechanics and its Applications, 493:421-429.

Liberatore, F., Scaparra, M. P., and Daskin, M. S. (2012). Hedging against disruptions with ripple effects in location analysis. Omega, 40(1):21-30.

Losada, C., Scaparra, M. P., Church, R. L., and Daskin, M. S. (2012). The stochastic inter- 
diction median problem with disruption intensity levels. Annals of Operations Research, 201(1):345-365.

Luathep, P., Sumalee, A., Ho, H. W., and Kurauchi, F. (2011). Large-scale road network vulnerability analysis: a sensitivity analysis based approach. Transportation, 38(5):799-817.

Lunday, B. J. and Sherali, H. D. (2012). Minimizing the maximum network flow: models and algorithms with resource synergy considerations. Journal of the Operational Research Society, 63:1693-1707.

Martel, A., Benmoussa, A., Chouinard, M., Klibi, W., and Kettani, O. (2013). Designing global supply networks for conflict or disaster support: the case of the Canadian Armed Forces. Journal of the Operational Research Society, 64:577-596.

Mattsson, L.-G. and Jenelius, E. (2015). Vulnerability and resilience of transport systems-a discussion of recent research. Transportation Research Part A: Policy and Practice, 81:1634 .

Murray, A. T., Matisziw, T. C., and Grubesic, T. H. (2007). Critical network infrastructure analysis: interdiction and system flow. Journal of Geographical Systems, 9(2):103-117.

Myung, Y. S. and Kim, H. J. (2004). A cutting plane algorithm for computing k-edge survivability of a network. European Journal of Operational Research, 156(3):579-589.

NASA. Climate Change: Vital Signs of the Planet. Avaliable at: https://climate.nasa.gov/effects Last time accessed: 2018-08-10.

Poorzahedy, H. and Abulghasemi, F. (2005). Application of Ant System to network design problem. Transportation, 32(3):251-273.

Poorzahedy, H. and Turnquist, M. A. (1982). Approximate algorithms for the discrete network design problem. Transportation Research Part B: Methodological, 16(1):45-55.

Rad, M. A. and Kakhki, H. T. (2017). Two Extended Formulations for Cardinality Maximum Flow Network Interdiction Problem. Networks, 69(4):367-377.

Richard Wollmer (1964). Removing Arcs from a Network. Operations Research, 12(6):934-940.

Scott, D. M., Novak, D. C., Aultman-Hall, L., and Guo, F. (2006). Network Robustness Index: A new method for identifying critical links and evaluating the performance of transportation networks. Journal of Transport Geography, 14(3):215-227.

Sheffi, Y. (1985). Urban Transportation Network. Englewood Cliffs, NJ.

Sohn, J. (2006). Evaluating the significance of highway network links under the flood damage: An accessibility approach. Transportation Research Part A: Policy and Practice, 40(6):491506.

Soleimani-Alyar, M. and Ghaffari-Hadigheh, A. (2018). Dynamic Network Interdiction Problem with Uncertain Data. International Journal of Uncertainty, Fuzziness and KnowledgeBased Systems, 26(02):327-342.

Starita, S., Amideo, A. E., and Scaparra, M. P. (2017a). Assessing Urban Rail Transit Systems Vulnerability: Metrics vs. Interdiction Models. In International Conference on Critical Information Infrastructures Security, pages 144-155. Springer.

Starita, S. and Scaparra, M. P. (2016). Optimizing dynamic investment decisions for railway systems protection. European Journal of Operational Research, 248(2):543-557.

Starita, S. and Scaparra, M. P. (2018). Passenger railway network protection: a model with variable post-disruption demand service. Journal of the Operational Research Society, 69(4):603-618.

Starita, S., Scaparra, M. P., and O'Hanley, J. R. (2017b). A dynamic model for road protection against flooding. Journal of the Operational Research Society, 68(1):74-88.

Sullivan, J., Novak, D., Aultman-Hall, L., and Scott, D. (2010). Identifying critical road segments and measuring system-wide robustness in transportation networks with isolating links: A link-based capacity-reduction approach. Transportation Research Part A: Policy and Practice, 44(5):323-336.

Sun, L., Huang, Y., Chen, Y., and Yao, L. (2018). Vulnerability assessment of urban rail transit based on multi-static weighted method in Beijing, China. Transportation Research Part A: Policy and Practice, 108:12-24.

Taylor, M. A., Sekhar, S. V., and D'Este, G. M. (2006). Application of Accessibility Based 
Methods for Vulnerability Analysis of Strategic Road Networks. Network and Spatial Economics, (6):267-291.

Taylor, M. A. and Susilawati (2012). Remoteness and accessibility in the vulnerability analysis of regional road networks. Transportation Research Part A: Policy and Practice, 46(5):761771.

Tran, T. H., O'Hanley, J. R., and Scaparra, M. P. (2017). Reliable Hub Network Design: Formulation and Solution Techniques. Transportation Science, 51(1):358-375.

Ukkusuri, S. V., Mathew, T. V., and Waller, S. T. (2007). Robust transportation network design under demand uncertainty. Computer-Aided Civil and Infrastructure Engineering, $22(1): 6-18$.

Wang, J. W. and Rong, L. L. (2009). Cascade-based attack vulnerability on the US power grid. Safety Science, 47(10):1332-1336.

Wardrop, J. G. (1952). Some theoretical aspects of road traffic research. Proceedings of the institution of civil engineers, 1(3):325-362.

Watling, D. and Balijepalli, N. (2012). A method to assess demand growth vulnerability of travel times on road network links. Transportation Research Part A: Policy and Practice, 46(5):772-789.

Wei, X., Zhu, C., Xiao, K., Yin, Q., and Zha, Y. (2018). Shortest Path Network Interdiction With Goal Threshold. IEEE Access, 6:29332-29343.

Wiesemann, W., Tsoukalas, A., Kleniati, P.-M., and Rustem, B. (2013). Pessimistic bilevel optimization. SIAM Journal on Optimization, 23(1):353-380.

Wood, R. K. (1993). Deterministic Network Interdiction. Mathl. Comput. Modelling, 17(2).

Yahoo News (2016). Cabbies block roads as france hit by multiple strikes. (Accessed on 08/06/2019).

Yin, H. Y. and Xu, L. Q. (2010). Measuring the Structural Vulnerability of Road Network: A Network Efficiency Perspective. J. Shanghai Jiaotong Univ. (Sci.), (6):736-742.

Zhang, L., de Gier, J., and Garoni, T. M. (2014). Traffic disruption and recovery in road networks. Physica A: Statistical Mechanics and its Applications, 401:82-102.

Zhang, X., Zheng, Z., Zhang, S., and Du, W. (2016). Partial interdiction median models for multi-sourcing supply systems. The International Journal of Advanced Manufacturing Technology, 84(1-4):165-181. 
Methods for Vulnerability Analysis of Strategic Road Networks. Network and Spatial Economics, (6):267-291.

Taylor, M. A. and Susilawati (2012). Remoteness and accessibility in the vulnerability analysis of regional road networks. Transportation Research Part A: Policy and Practice, 46(5):761771.

Tran, T. H., O'Hanley, J. R., and Scaparra, M. P. (2017). Reliable Hub Network Design: Formulation and Solution Techniques. Transportation Science, 51(1):358-375.

Ukkusuri, S. V., Mathew, T. V., and Waller, S. T. (2007). Robust transportation network design under demand uncertainty. Computer-Aided Civil and Infrastructure Engineering, $22(1): 6-18$.

Wang, J. W. and Rong, L. L. (2009). Cascade-based attack vulnerability on the US power grid. Safety Science, 47(10):1332-1336.

Wardrop, J. G. (1952). Some theoretical aspects of road traffic research. Proceedings of the institution of civil engineers, 1(3):325-362.

Watling, D. and Balijepalli, N. (2012). A method to assess demand growth vulnerability of travel times on road network links. Transportation Research Part A: Policy and Practice, 46(5):772-789.

Wei, X., Zhu, C., Xiao, K., Yin, Q., and Zha, Y. (2018). Shortest Path Network Interdiction With Goal Threshold. IEEE Access, 6:29332-29343.

Wiesemann, W., Tsoukalas, A., Kleniati, P.-M., and Rustem, B. (2013). Pessimistic bilevel optimization. SIAM Journal on Optimization, 23(1):353-380.

Wood, R. K. (1993). Deterministic Network Interdiction. Mathl. Comput. Modelling, 17(2).

Yahoo News (2016). Cabbies block roads as france hit by multiple strikes. (Accessed on 08/06/2019).

Yin, H. Y. and Xu, L. Q. (2010). Measuring the Structural Vulnerability of Road Network: A Network Efficiency Perspective. J. Shanghai Jiaotong Univ. (Sci.), (6):736-742.

Zhang, L., de Gier, J., and Garoni, T. M. (2014). Traffic disruption and recovery in road networks. Physica A: Statistical Mechanics and its Applications, 401:82-102.

Zhang, X., Zheng, Z., Zhang, S., and Du, W. (2016). Partial interdiction median models for multi-sourcing supply systems. The International Journal of Advanced Manufacturing Technology, 84(1-4):165-181. 